\title{
A model-based approach to disentangling facilitation and interference effects in conflict tasks
}

\author{
Nathan J. Evans ${ }^{a}$ and Mathieu Servant ${ }^{b}$ \\ ${ }^{a}$ School of Psychology, University of Queensland, Australia \\ ${ }^{b}$ Laboratoire de Psychologie, Université de Franche-Comté, France
}

Word count: 12,494

Keywords:

Conflict diffusion models — Facilitation effects - Interference effects — Probability density approximation — Flanker task — Simon task

Correspondence concerning this article may be addressed to: nathan.j.evans@uon.edu.au (NJE) or mathieu.servant@univ-fcomte.fr (MS)

NJE was supported by an Australian Research Council Discovery Early Career Researcher Award (DE200101130). MS was supported by an AENER grant from the Bourgogne Franche-Comté region. 


\begin{abstract}
Conflict tasks have become one of the most dominant paradigms within cognitive psychology, with their key finding being the conflict effect: that participants are slower and less accurate when task-irrelevant information conflicts with task-relevant information (i.e., incompatible trials), compared to when these sources of information are consistent (i.e., compatible trials). However, the conflict effect can consist of two separate effects: facilitation effects, which is the amount of benefit provided by consistent task-irrelevant information, and interference effects, which is the amount of impairment caused by conflicting task-irrelevant information. While previous studies have attempted to disentangle these effects using neutral trials, which contrast compatible and incompatible trials to trials that are designed to have neutral task-irrelevant information, these analyses rely on the assumptions of Donder's subtractive method, which are difficult to verify and may be violated in some circumstances. Here, we develop a model-based approach for disentangling facilitation and interference effects, which extends the existing diffusion model for conflict tasks (DMC) framework to allow for different levels of automatic activation in compatible and incompatible trials. Comprehensive parameter recovery assessments display the robust measurement properties of our model-based approach, which we apply to 9 previous data sets from the flanker (6) and Simon (3) tasks. Our findings suggest asymmetric facilitation and interference effects, where interference effects appear to be present for most participants across most studies, whereas facilitation effects appear to be small or non-existent. We believe that our novel model-based approach provides an important step forward for understanding how information processing operates in conflict tasks, allowing researchers to assess the convergence or divergence between experimental-based (i.e., neutral trials) and model-based approaches when investigating facilitation and interference effects.
\end{abstract}




\section{Introduction}

The cornerstone of the cognitive approach to explaining human behaviour has been attempting to understand how people process incoming information in a range of different contexts (Tversky \& Kahneman, 1974; Ratcliff, 1978; Kahneman \& Tversky, 1979; Roitman \& Shadlen, 2002; Purcell et al., 2010; Hawkins, Forstmann, Wagenmakers, Ratcliff, \& Brown, 2015; B. A. Eriksen \& Eriksen, 1974; Simon \& Small Jr., 1969; Stroop, 1935). One of the most commonly explored contexts has been situations where multiple, potentially conflicting sources of information are available, some of which are relevant to the task at hand, and some of which are irrelevant. This context is commonly instantiated in laboratory settings through conflict tasks, where participants are presented with decisionrelevant and decision-irrelevant information, with the different sources of information either directing participants towards the same response (i.e., consistent information) or different responses (i.e., conflicting information). Previous research has involved the design and investigation of a range of different conflict tasks, with the most notable tasks being the

flanker (B. A. Eriksen \& Eriksen, 1974), Simon (Simon \& Small Jr., 1969), and Stroop (Stroop, 1935), and the general finding being a conflict effect: that participants are slower and less accurate at the task when the task-irrelevant information is conflicting with the task-relevant information (i.e., "incompatible trials"), compared to when these sources of information are consistent with one another (i.e., "compatible trials"). The conflict effect is commonly interpreted through the dual-route framework (Kornblum, Hasbroucq, \& Osman, 1990; Ridderinkhof, van der Molen, \& Bashore, 1995; Ridderinkhof, 2002; Hommel, 1993; De Jong, Liang, \& Lauber, 1994), which proposes that relevant and irrelevant information are each processed through different parallel routes (a controlled and automatic route, respectively) that generally converge within the decision process (though see van den Wildenberg et al., 2010). Importantly, the controlled and automatic routes activate the 
same response in compatible trials and opposing responses in incompatible trials, suggesting that conflict effects are the result of both the irrelevant information in incompatible trials interfering with processing the relevant information, and the consistent irrelevant information in compatible trials facilitating processing of the relevant information.

Although the primary focus of conflict tasks is the conflict effect, the conflict effect can thus be thought of as the combination of two theoretically separable effects: facilitation and interference effects (Craft \& Simon, 1970; Simon \& Craft, 1970). Specifically, facilitation effects refer to the amount of benefit provided by consistent task-irrelevant information in compatible trials, and interference effects refer to the amount of impairment caused by conflicting task-irrelevant information in incompatible trials, which can each cause the conflict effect observed between compatible and incompatible trials. Facilitation and interference effects also have important implications for theories of conflict processing. For example, dual-route theories assume that the controlled and automatic processing routes are independent, and therefore, the amount of automatic activation only depends on the irrelevant information, which must be identical in compatible and incompatible trials (Kornblum et al., 1990). However, it is also possible that the conflict effect is the result of unequal amounts of facilitation and interference, or potentially even facilitation or interference alone (Umiltà, Rubichi, \& Nicoletti, 1999; Simon \& Small Jr., 1969; Craft \& Simon, 1970; C. W. Eriksen \& Eriksen, 1979; Yeh \& Eriksen, 1984; C. W. Eriksen \& St James, 1986). An unequal amount of facilitation and interference, or a complete absence of one effect, would suggest that certain theoretical assumptions about the process underlying conflict effects are incorrect, such as the assumption of independence between the controlled and automatic processing routes in dual-route theories.

Previous research efforts focused on assessing facilitation and interference have adopted experimental-based approaches to separating these effects. Specifically, these 
experimental-based approaches involve the addition of neutral trials, which intend to provide a pure measurement of the information processing of task-relevant information in conflict tasks (Umiltà et al., 1999; Simon \& Small Jr., 1969; Craft \& Simon, 1970; C. W. Eriksen \& Eriksen, 1979; Yeh \& Eriksen, 1984; C. W. Eriksen \& St James, 1986; Simon \& Craft, 1970; Wallace, 1971; Miller, 1991; T. L. Brown, 2011; Burle, Possamaï, Vidal, Bonnet, \& Hasbroucq, 2002). As facilitation and interference effects are both the result of task-irrelevant information influencing the processing of task-relevant information, trials where the task-irrelevant information has no influence on the processing of task-relevant information would allow for a pure measurement of task-relevant information processing. Neutral trials provide neutral task-irrelevant information, which is assumed to not direct participants towards any response alternative, meaning that the difference in performance between compatible and neutral trials should provide a measurement of facilitation effects, and the difference in performance between neutral and incompatible trials should provide a measurement of interference effects. Neutral trials are also a theoretically agnostic method for distinguishing between facilitation and interference effects, meaning that the use of neutral trials does not require a commitment to any specific theory of conflict processing, making them a preferable method for researchers who do not wish to make assumptions about the underlying process. The use of neutral trials has proved quite popular within the conflict literature, with many studies implementing these trials in an attempt to distinguish between facilitation and interference effects in the flanker (B. A. Eriksen \& Eriksen, 1974; Miller, 1991), Simon (Simon \& Small Jr., 1969; Craft \& Simon, 1970; Wallace, 1971), and Stroop (T. L. Brown, 2011) tasks.

However, the conclusions drawn from the use of neutral trials have been mixed ${ }^{1}$.

\footnotetext{
${ }^{1}$ As the empirical focus of the current study is on the flanker and Simon tasks, our discussion of the previous literature on neutral trials focuses on these tasks. However, it should be noted that neutral trials are common within other conflict tasks, such as the Stroop (T. L. Brown, 2011).
} 
In the Simon task, neutral trials are usually implemented by presenting the target at a location equidistant from the two responses (usually the vertical meridian). The results from these experiments have generally provided roughly equal facilitation and interference effects, with the absolute difference in response time from neutral trials being roughly equal for compatible and incompatible trials (Burle et al., 2002; Wallace, 1971). However, in some of these cases, the magnitude of the facilitation effect appeared much smaller than the magnitude of the interference effect. Umiltà et al. (1999) provided evidence that the relative magnitude of facilitation and interference in the Simon task is in fact mediated by visual acuity. When neutral targets are presented at the center of the screen, processing benefits from a visual acuity advantage, resulting in facilitation effects that are smaller in magnitude than interference effects. However, when neutral, compatible, and incompatible targets are equidistant from the central fixation point (forming an equilateral triangle and thus controlling for differences in visual acuity), the magnitude of facilitation and interference effects is roughly similar. Conversely, there are some studies in the literature that have shown no facilitation effects in the Simon task, and that cannot be explained by differences in visual acuity. Simon and Small Jr. (1969), for example, designed an auditory version of the Simon task, in which subjects had to press a left or a right button depending on the frequency (high vs low) of a tone stimulus presented monaurally (see Craft \& Simon, 1970, for a visual variant of this task with similar findings). In the neutral condition, the tone stimulus was presented binaurally. The results showed slightly faster response times in neutral compared to compatible trials. It has been argued that the uncertainty with regards to the location of the target in monaural trials may have slowed down response times compared to the binaural presentation (Wallace, 1971). In addition, monaural and binaural conditions were blocked, which may have produced a shift in decision criterion. This brief review of the literature illustrates the difficulty in identifying a pure neutral 
condition.

In the flanker task, neutral trials are usually implemented in one of two different ways. The first way consists of presenting no flanker at all. One issue with this method is that neutral trials are usually faster than compatible trials when the spacing between letters is small (B. A. Eriksen \& Eriksen, 1974). This is because adjacent flankers generate interference at the stimulus input level presumably due to low-level contour interactions, a phenomenon known as foveal crowding (Lev, Yehezkel, \& Polat, 2014). The second way consists of presenting flanker stimuli that are neutral with respect to the response feature. One issue with this method is that flankers can still share feature similarities with members of the stimulus set. Eriksen and colleagues (C. W. Eriksen \& Eriksen, 1979; Yeh \& Eriksen, 1984; C. W. Eriksen \& St James, 1986) showed that neutral flankers that share similarities with the target produce little effect on RT. By contrast, neutral flankers that share similarities with the competing response stimulus usually increase RT, though to a lesser extent than incompatible flankers. These findings again illustrate the difficulty in identifying a pure neutral condition.

Neutral trials undoubtedly provide a useful experimental-based approach for distinguishing between facilitation and interference effects in conflict tasks. However, like any scientific approach, the validity of neutral trials is dependent on specific assumptions, and if these assumptions are incorrect, then the conclusions drawn from neutral trials about facilitation and interference effects could be misleading. Broadly speaking, the use of neutral trials in conflict tasks can be defined as a type of cost-benefit analysis (Posner \& Snyder, 1975; Beller, 1971; Meyer \& Schvaneveldt, 1971), where the researcher is interested in determining the cost of an invalid cue (i.e., the conflicting task-irrelevant information in an incompatible trial), and the benefit of a valid cue (i.e., the task-relevant information in a compatible trial). As discussed in detail by Jonides and Mack (1984), while cost-benefit 
analyses are common within the psychological literature, they rely on the same, strict rationale as Donder's subtractive method (Donders, 1969). Specifically, in order for neutral trials to accurately separate costs from benefits, the neutral cue must (1) be completely uninformative, in the sense that it does not provide any form of cue towards either response alternative, and (2) differ from valid and invalid cues in only the informativeness. Importantly, we believe it is intuitively plausible that these assumptions could be violated in the flanker and/or Simon tasks. For example, in the flanker task, it is possible that some perceptual features of neutral flankers could still activate a response, through some of the perceptual features of the neutral flankers being shared with one of the response options (B. A. Eriksen \& Eriksen, 1974). In the Simon task, it is possible that the exact location of the neutral stimulus could still create other sources of facilitation and interference. For example, a central presentation may facilitate processing due to visual acuity (Umiltà et al., 1999). Importantly, any violations of the strict rationale of Donder's subtractive method could result in inferences on neutral trials that are misleading, as they would not serve as a pure subtraction from cued trials.

There are alternatives to experimental-based approaches for gaining insight into underlying cognitive processes. Within mathematical psychology, researchers often use mathematical models, which serve as formalized representations of theories about the underlying cognitive process of interest (Stone, 1960; Shiffrin \& Steyvers, 1997; Usher \& McClelland, 2001; Servant, White, Montagnini, \& Burle, 2015; Osth \& Dennis, 2015; Evans, Brown, Mewhort, \& Heathcote, 2018; Evans, Dutilh, Wagenmakers, \& van der Maas, 2020). Importantly, these models can decompose observed data into estimates of the components of the underlying cognitive process, by assessing the empirical data under the constraints provided by the assumptions of the theory (i.e., model application; Crüwell, Stefan, \& Evans, 2019). These estimates often provide insight into empirical questions about the process of 
interest, allowing researchers to answer these questions without the need for novel experimental paradigms or conditions, or the specific assumptions underlying approaches such as Donder's subtractive method. For example, a well-established effect within the ageing literature is that older adults perform cognitive tasks more slowly than younger adults. The long-held explanation for this was a "cognitive slowdown" (Salthouse, 1996), where the mental processing speed of adults decreases as they age, making older adults slower at cognitive tasks. However Ratcliff, Thapar, and McKoon (2001) were able to use a cognitive model - the diffusion model (Ratcliff, 1978; Ratcliff \& Rouder, 1998) - to decompose the choice response time distributions into parameters that reflected mental processing speed, response caution, and motor response speed. Their findings indicated that older adults were more cautious and had slower motor responding than younger adults, but did not appear to have slower mental processing speed. This shows how model-based approaches can be used to provide novel insights into research questions, and how their insights can differ from those of experimental-based approaches.

The current study aims to develop a novel model-based approach to disentangling facilitation and inference effects in conflict tasks. As discussed above, model-based approaches provide an alternative to experimental-based approaches, replacing the assumptions about the relationship between experimental conditions in experimental-based approaches with assumptions contained within the model based on psychological theory. However, no model-based approaches currently exist for separating facilitation and interference effects in conflict tasks. The remainder of our article will take the following structure. First, we provide a review of the existing models of conflict tasks that can account for the entire response time distributions - known as the conflict diffusion models (Hübner, Steinhauser, \& Lehle, 2010; White, Ratcliff, \& Starns, 2011; Ulrich, Schröter, Leuthold, \& Birngruber, 2015) - and whether these models can theoretically be extended 
to disentangling facilitation and interference effects. Second, we provide an extension of one of these models - the diffusion model for conflict tasks (DMC; Ulrich et al., 2015) that can theoretically distinguish between facilitation and interference effects, and assess whether it can reliably distinguish between these effects in practical settings. Third, we apply our validated novel framework to 9 conflict task data sets (6 flanker, 3 Simon), with our findings suggesting an absence of facilitation and presence of interference across most participants in most experiments, providing model-based evidence that the observed conflict effects may be mostly composed of interference effects. We also wish to note that our aim is not to claim that model-based approaches are superior to experimental-based approaches, or that model-based approaches should be used instead of experimental-based approaches to assess facilitation and interference effects in conflict tasks. Rather, our aim is to provide a model-based approach for disentangling facilitation and interference effects in conflict tasks, which we hope can serve as a complementary method to existing experimental-based approaches, allowing researchers to provide converging - or diverging - assessments of these effects using different methods that rely on different assumptions.

\section{Model Development}

\section{Review of existing models}

Within the area of decision-making, the diffusion model (Stone, 1960; Ratcliff, 1978; Ratcliff \& Rouder, 1998; see Evans \& Wagenmakers, 2020 for a review) has become the dominant mathematical model of human decision-making, proposing that information accumulates over time for the different decision alternatives in the form of evidence, until the evidence for one alternative reaches some threshold level, and a decision is triggered. Importantly, these theoretical assumptions allow the diffusion model to decompose the observed choice response time distributions into estimates of the decision-making process, 
such as the rate of evidence accumulation for the correct alternative over the incorrect alternative, known as the "drift rate", the amount of evidence required to trigger a decision, known as the "decision threshold", and the amount of time dedicated to encoding and motor processes unrelated to the decision, known as the "non-decision time". More formally, the diffusion model can be written as a stochastic differential equation:

$$
d x=v d t+c d W
$$

where $x$ is the evidence, $W$ is the Wiener process (noise from the standard normal distribution that evolves with the square root of time), and $c$ is the diffusion coefficient, with $c$ being fixed to 0.1 to solve a scaling property within the model. The ability to estimate these components of the decision-making process has made the diffusion model one of the most useful tools within cognitive psychology, being used to address questions in areas such as such as intelligence (van Ravenzwaaij, Brown, \& Wagenmakers, 2011; Lerche et al., 2020), performance optimality (Starns \& Ratcliff, 2012; Evans \& Brown, 2017; Evans, Bennett, \& Brown, 2019), cognitive workload (Ratcliff \& Strayer, 2014; Tillman, Strayer, Eidels, \& Heathcote, 2017; Howard, Evans, Innes, Brown, \& Eidels, 2020), ageing (Ratcliff et al., 2001; Servant \& Evans, 2020), emotion processing (Lerche, Bucher, \& Voss, 2019), alcohol consumption (van Ravenzwaaij, Dutilh, \& Wagenmakers, 2012), and ego depletion (Lin, Saunders, Friese, Evans, \& Inzlicht, 2020), just to name a few.

One assumption of the standard diffusion model is that the rate of evidence accumulation is constant over time, as it assumes that decision formation involves integrating many noisy samples of the same, task-relevant information. Importantly, this assumption means that the diffusion model is invalid for conflict tasks, as conflict tasks involve both task-relevant and task-irrelevant sources of information, with empirical evidence suggesting that the impact of task-irrelevant information on decision formation decreases over time 
(e.g., Hommel, 1993, 1994; Ridderinkhof, 2002; Mattler, 2003). To address this limitation, several extensions of the diffusion framework have been developed for conflict tasks - the shrinking spotlight model (SSP), the dual-stage two-phase model (DSTP), and the diffusion model for conflict tasks (DMC) - which each involve a mechanism for incorporating task-relevant and task-irrelevant sources of information through either visual attention (Hübner et al., 2010; White et al., 2011) or automaticity (Ulrich et al., 2015). These conflict diffusion models are each able to explain the choice response time distributions observed in the flanker task (Hübner et al., 2010; White et al., 2011; Ulrich et al., 2015), though to varying degrees (Evans \& Servant, 2020), and only the DSTP and DMC being able to extend to other conflict tasks (Ulrich et al., 2015; Hübner \& Töbel, 2019). Here, we describe the dynamics of each of these models, and whether they can be extended to provide a model-based approach for disentangling facilitation and interference.

Shrinking Spotlight Model. The SSP (White et al., 2011; Figure 1) is based on theories of spatial visual attention, and has been specifically developed to account for behavioral performance in the flanker task. SSP proposes that the flanker effect is the result of a gradually narrowing attentional spotlight. The attentional spotlight reflects both the region of physical space that attention is focused on and where attention is most heavily concentrated, with all stimuli inside the spotlight being processed, and those where attention is more heavily concentrated contributing more information. The spotlight is initially broad, incorporating information from both the target and flankers, and then narrows over time towards the target, eventually filtering out the information provided by the flankers. Formally, the SSP can be written as a stochastic differential equation with a time-varying drift rate:

$$
d x(t)=v(t) d t+c d W
$$


where $v(t)$ is the drift rate at time $t$. The time-varying drift rate is determined by the attentional spotlight, which changes over time, and perceptual strength of the stimuli, which is constant over time:

$$
v(t)=s_{t a}(t) \times p_{t}+s_{f l}(t) \times p_{f l}
$$

where $s_{t a}(t)$ and $s_{f l}(t)$ is the amount of attention allocated to the target and flankers, respectively, at time $t$, and $p_{t}$ and $p_{f l}$ is the perceptual strength of the target and flankers, respectively. Note that $p_{f l}$ is positive for compatible trials and negative for incompatible trials, which reflects the direction of the flanker input. The spotlight is represented by a normal distribution with mean 0 (i.e., centred on the target) and standard deviation $S D(t)$, with the shrinking mechanism being captured by a standard deviation that decreases over time:

$$
S D(t)=S D_{0}-S D_{r} \times t
$$

where $S D_{0}$ is the initial standard deviation of the spotlight and $S D_{r}$ is the rate of decrease. Note that standard deviation of the spotlight has a minimum value of 0.001 , which is assumed to reflect the maximum focus. For each time step $t$, the amount of attention allocated to the target $\left(s_{t a}(t)\right)$ and flankers $\left(s_{f l}(t)\right)$ is determined by the cumulative density of the normal distribution within the area of the target, with the target assumed to be one unit of area:

$$
\begin{array}{r}
s_{t a}(t)=\int_{-0.5}^{0.5} \Phi(x \mid 0, S D(t)) d x \\
s_{f l}(t)=\int_{-\infty}^{-0.5} \Phi(x \mid 0, S D(t)) d x+\int_{0.5}^{\infty} \Phi(x \mid 0, S D(t)) d x
\end{array}
$$


where $\Phi$ is the cumulative density of the normal distribution at $x$, given the mean and standard deviation parameters of the normal distribution. SSP thus assumes that the amount of attentional ressources allocated to the task is constant across time and experimental conditions, regardless of how the attention is being distributed. Therefore, in situations where the perceptual strength is assumed to be the same for targets and flankers - a common assumption when applying the SSP (White et al., 2011; White, Servant, \& Logan, 2018; Evans \& Servant, 2020) - the drift rate in compatible trials is actually constant over time. Specifically, as both the target and flankers are providing evidence for the same alternative, the total amount of evidence accumulated at any one point in time is constant. This means that standard implementations of SSP implicitly assume a lack of facilitation in compatible trials, as processing the target with compatible flankers is identical to processing only the target (White et al., 2011). Furthermore, while time-varying drifts can technically be created in compatible trials by allowing the perceptual strength of the flankers $\left(p_{f l}\right)$ to differ from the perceptual strength of the target $\left(p_{t}\right)$, these time-varying drift rates will only display facilitation when the perceptual strength for the flankers is stronger than for the target (Figure 1). This assumption appears implausible given the reduced acuity in parafoveal vision (Strasburger, Rentschler, \& Jüttner, 2011; Staugaard, Petersen, \& Vangkilde, 2016).

To summarize, the ability of the SSP to capture flanker data lends credence to the hypothesis that there may be a lack of facilitation in the flanker task. However, there does not appear a plausible way for the model to incorporate facilitation effects, making it a poor tool for a model-based approach to disentangling facilitation and interference effects.

Dual-Stage Two-Phase Model. The DSTP (Hübner et al., 2010; Figure 1) is also based on theories of visual attention, and has proven to capture behavioral performance in both flanker (Hübner et al., 2010) and Simon tasks (Hübner \& Töbel, 2019). Contrary to 
the SSP, DTSP proposes a two phase account of selective attention and resulting conflict effects. The first phase consists of a dual-route framework, where two processes occur simultaneously: a response selection process and a stimulus selection process. In the first phase attention is spread broadly, and the response selection process is influenced by both task-relevant and task-irrelevant stimulus information. Formally, this is reflected by a standard diffusion process, where the drift rate $v_{p 1}$ is made up of two separate drift rates:

$$
v_{p 1}=v_{r}+v_{i}
$$

where $v_{r}$ is the drift rate for the task-relevant information and $v_{i}$ is the drift rate for the task-irrelevant information, with the latter being positive in compatible trials and negative in incompatible trials. The stimulus selection process is an unobserved decision about which item to focus attention on; in the case of the flanker task, this would be either the target, or one of the flankers. Formally, this is reflected by a standard diffusion process with a drift rate of $v_{s s}$, which is the drift rate in favour of correctly selecting the target. In compatible trials it does not matter which item is selected, as all items provide information favouring the same response alternative.

The second phase of response selection only occurs if the stimulus selection process finishes before the response selection process. If a stimulus is selected before a response, attention becomes focused on this selected stimulus, meaning that the response selection process is now only influenced by a single source of information $\left(v_{p 2}\right)$. Consequently, the response selection process can more generally be thought of as a piecewise diffusion process:

$$
v_{p 1}=v_{r}+v_{i} ; v_{p 2}=v_{p 2}
$$

were $v_{p 1}$ and $v_{p 2}$ correspond to the drift rate of the response selection process in phase 
1 and phase 2 respectively. The switch time is determined by the finishing time of the stimulus selection process, and $v_{p 2}$ is positive unless both the trial is incompatible and the task-irrelevant stimulus is selected.

The standard DSTP implementation assumes equal facilitation and interference effects, as the same absolute drift rate $\left(v_{i}\right)$ is used to reflect the task-irrelevant information in the first phase regardless of whether the trial is compatible or incompatible (Hübner et al., 2010). However, it is mathematically impossible to disentangle facilitation and interference effects in the standard DSTP framework. While $v_{i}$ can technically be separated into $v_{i, c o m p}$ and $v_{i, i n c o m p}$, this would result in three time-constant drift rates $-v_{r}, v_{i, c o m p}$, and $v_{i, \text { incomp }}$ - for two conditions - compatible and incompatible - meaning that the separate drift rates can take on any values and not change the predictions of the model, provided that they still combine to create the same overall drift rates in each condition (Figure 1). Therefore, while the DSTP is also a successful model, there does not appear to be a natural way for the model to identify differing levels of facilitation and interference, making it a poor tool for a model-based approach to disentangling facilitation and interference effects.

Diffusion Model for Conflict Tasks. In contrast to SSP and DSTP, the DMC (Ulrich et al., 2015; Figure 2) is based on theories of automaticity, and proposes that conflict effects are the result of an initial pulse of automatic response activation from the taskirrelevant information. DMC builds upon a dual-route framework - as with DSTP - but involves a time-varying drift rate - as with SSP. Specifically, the time-varying drift rate is determined by two processes: the automatic process, which changes over time, and controlled processing of task-relevant information, which is constant over time:

$$
v(t)=v_{c}+v_{a}(t)
$$


where $v_{c}$ is the drift rate of the controlled process, and $v_{a}(t)$ is the drift rate of the automatic process at time $t$. Note that $v_{a}(t)$ is positive for compatible trials and negative for incompatible trials, which reflects the alternative that the automatic activation is providing evidence towards. As automatic activation is theorized to be short-lived, the drift rate of the automatic process should be high when processing starts, and quickly decrease over time. To reflect this, $v_{a}(t)$ changes over time according to the first derivative of a rescaled gamma function, which produces a pulse of automatic activation with varying shape, amplitude and latency depending on the parameterisation:

$$
v_{a}(t)=\zeta \times e^{\frac{-t}{\tau}} \times\left[\frac{t \times e}{(\alpha-1) \times \tau}\right]^{\alpha-1} \times\left[\frac{\alpha-1}{t}-\frac{1}{\tau}\right]
$$

where $\zeta$ is the rescaling parameter - which is also the maximum amplitude of the automatic activation - and $\alpha$ and $\tau$ are the shape and scale of the gamma function, respectively. In all previous implementations of DMC (e.g., Ulrich et al., 2015; Servant \& Evans, 2020; Hedge, Vivian-Griffiths, Powell, Bompas, \& Sumner, 2019; Evans \& Servant, 2020; Mittelstädt \& Miller, 2018), all parameters of the automatic activation process have been constrained to take the same values over compatibility conditions, meaning that the standard DMC model implicitly assumes symmetric facilitation and interference effects. However, as we discuss in the next section, this assumption can be easily and plausibly relaxed, creating an extension of DMC that is able to provide a model-based method for disentangling facilitation and interference effects.

Extension of $D M C$

DMC is theoretically able to separate facilitation and interference effects through only a minor extension to the existing definition. Specifically, we propose allowing the $\zeta$ parameter of the model - the scaling of the gamma function, which determines the overall 
amount of automatic activation - to vary between compatible and incompatible trials (see Figure 2). Formally, this can be reflected by a slight adjustment to the calculation of the automatic drift rate:

$$
\begin{gathered}
v_{a, \text { comp }}(t)=\zeta_{\text {comp }} \times e^{\frac{-t}{\tau}} \times\left[\frac{t \times e}{(\alpha-1) \times \tau}\right]^{\alpha-1} \times\left[\frac{\alpha-1}{t}-\frac{1}{\tau}\right] \\
v_{a, \text { incomp }}(t)=\zeta_{\text {incomp }} \times e^{\frac{-t}{\tau}} \times\left[\frac{t \times e}{(\alpha-1) \times \tau}\right]^{\alpha-1} \times\left[\frac{\alpha-1}{t}-\frac{1}{\tau}\right]
\end{gathered}
$$

where $\zeta_{c o m p}$ is the rescaling parameter for compatible trials and $\zeta_{\text {incomp }}$ is the rescaling parameter for incompatible trials. Values of $\zeta_{\text {comp }}$ that are close to zero suggest a lack of facilitation, whereas values of $\zeta_{\text {incomp }}$ that are close to zero suggest a lack of interference. Furthermore, similar values of $\zeta_{c o m p}$ and $\zeta_{\text {incomp }}$ suggest equal facilitation and interference effects, with the extended model reducing to the standard DMC when $\zeta_{\text {comp }}=\zeta_{\text {incomp }}$. Unlike DSTP, the time-varying source of task-irrelevant information in DMC - when constrained to take on a specific pulse-like function, such as the first derivative of the gamma function - provides a mathematical separation between the different summed drift rates, as changes in the automatic process strongly influence the early parts of the trial and disappear by the later stages of the trial, whereas changes in the controlled process are constant across the entire trial. Therefore, adjusting the $\zeta_{\text {comp }}$ parameter cannot be counteracted by equivalent changes in the $v_{c}$ parameter and the $\zeta_{\text {incomp }}$ parameter.

While our approach provides a simple model-based method of separately measuring facilitation and interference effects, some readers may wonder about the theoretical plausibility of our general assumption that the level of automatic activation can be different between compatible and incompatible trials. Indeed, following general assumptions of the dual-route model framework, facilitation and interference effects should be symmetric, as 
the routes are thought to be independent of one another, and so the processing of taskirrelevant information should not depend on the task-relevant information (e.g., Kornblum et al., 1990). However, there are two important points to note here. First, dual-route models commonly assume that the two routes converge at the response selection stage, and therefore, it seems likely that the exact process of convergence could determine whether or not facilitation and/or interference occurs. Second, while DMC builds upon a dual-route framework, it is agnostic with respect to the source and mechanisms giving rise to the automatic activation, meaning that the automatic activation could be the result of both task-relevant and task-irrelevant information. For example, if the automatic activation was a result of unfocused visual attention - similar to the early stages of SSP and DSTP - then it seems plausible that the automatic activation is influenced by both task-relevant and task-irrelevant information, and that facilitation and interference could be asymmetric, as in SSP.

Here, we provide the precise definition for our extension, which we implement throughout the remainder of our study. As with the standard diffusion model and DMC implementation, our extension includes parameters for decision thresholds $( \pm a)$ and nondecision time $\left(t_{e r}\right)$ components of the process, with the diffusion coefficient fixed to 0.1 . As with the standard DMC, the drift rate is split into a controlled process and an automatic process, with a single parameter for the controlled process $\left(v_{c}\right)$, and a two parameter gamma function for the automatic process $(\alpha, \tau)$ that is separately rescaled in the compatible and incompatible conditions $\left(\zeta_{\text {comp }}, \zeta_{\text {incomp }}\right)$. In line with Ulrich et al. $(2015)$, we fix the shape parameter $(\alpha)$ at the value of 2 , which (1) removes a potential source of sloppiness in the model (see Holmes, 2015; Gutenkunst et al., 2007, for the "sloppy models" problem), and (2) provides a clearer theoretical interpretation for $\tau$, as with this fix $\tau$ corresponds to the peak latency of the automatic activation. The standard diffusion model often also includes 
a fourth parameter, the starting point $(z)$, which is the starting amount of evidence and reflects a-priori bias for one response option over another. However, for parsimony we set $z$ to be the midpoint between the two decision thresholds, similar to previous applications of DMC (Ulrich et al., 2015; Servant, White, Montagnini, \& Burle, 2016; Hedge et al., 2019). It should also be noted that the diffusion framework often includes three additional parameters for the between-trial variability in drift rate, starting point, and non-decision time, respectively (Ratcliff, 1978; Ratcliff \& Rouder, 1998; Ratcliff \& Tuerlinckx, 2002), and that these parameters can be integrated into the DMC framework (Evans \& Servant, 2020). However, these between-trial variability parameters can compromise the measurement properties of the model (van Ravenzwaaij \& Oberauer, 2009; Lerche \& Voss, 2016; Lerche, Voss, \& Nagler, 2017; Boehm et al., 2018; Evans, Tillman, \& Wagenmakers, 2020), which would prevent us from robustly disentangling facilitation and interference effects.

\section{Assessment of DMC extension}

Our primary aim is to use our extension of the DMC framework as a measurement tool for distinguishing between facilitation and interference effects in conflict tasks. Therefore, the following assessment focuses on testing the measurement properties of our DMC extension (see White et al., 2018 and Hübner \& Pelzer, 2020 for an assessment of the measurement properties of the standard DMC and other conflict models), in order to ensure that the parameters relating to facilitation and interference effects are distinguishable from one another in practical sample sizes. Specifically, we perform a comprehensive parameter recovery study, which involves generating a large number of simulated data sets from the model and then fitting the model to each of these data sets, in order to assess whether the estimated parameter values match the generating parameter values. Importantly, successful parameter recovery reflects the minimum standard for reliable inference on model parameters, and should always be conducted when developing a new model for use as a 
measurement tool (see Evans, 2020a for a discussion and example of the issues caused by incomplete initial parameter recovery assessments).

To select the generating sets of parameter values, we used a Latin hypercube sampling design, which provides the most efficient method for sampling from a multi-dimensional continuous space (McKay, Beckman, \& Conover, 1979; Evans, Trueblood, \& Holmes, 2020; Evans, 2020a). We sampled 3,000 parameter sets from the hypercube in the first simulation study, and 10,000 in the other simulation studies due to the wider range of parameters, generating a single data set from each parameter set. We assessed whether each generated data set resembled a realistic response time distribution, in order to ensure that our recovery assessment only included parameter sets that produce plausible predictions for empirical data. A data set had to meet all inclusion criteria to be included, with the inclusion criteria being:

$$
\begin{gathered}
0.4<\text { Mean RT }<2.5 \\
0.4<\text { Median RT }<2.5 \\
0.1<\text { IQR of RT }<2 \\
\text { Minimum RT }<1 \\
0.5<\text { Maximum RT }<5 \\
0.6<\text { Accuracy }<1
\end{gathered}
$$

These inclusion criteria results in $1,400,1,664,1,840$, and 1,955 data sets being included for each simulation study, respectively.

We fit our DMC extension using Bayesian parameter estimation, which attempts to estimate the probability of each potential parameter value after having observed the em- 
pirical data (i.e., the posterior distribution). Specifically, we estimated the posterior distributions using Differential Evolution Markov Chain Monte Carlo (DE-MCMC; Ter Braak, 2006; Turner, Sederberg, Brown, \& Steyvers, 2013), with $3 k$ parallel chains - where $k$ is the number of free parameters in the model - each sampling for 3,500 iterations, with the first 2,250 iterations from each chain discarded as burn-in. As analytic solutions for the likelihood function of time-varying models are not possible, and numeric solution are extremely computationally taxing to calculate (Smith, 2000), we approximated the likelihood function using probability density approximation (PDA; Turner \& Sederberg, 2014; Holmes, 2015). PDA provides an approximation to the likelihood function of a model by fitting a density kernel to the simulated predictions of the model. We used 10,000 simulated predictions per condition to create the density kernel for each likelihood calculation, and simulated the model using the Euler's method (e.g., S. D. Brown, Ratcliff, \& Smith, 2006) with a step-size of 0.01s (Evans \& Servant, 2020). The specific random number generation method and simulation framework was based on the method and framework of Evans (2019). Our extension of DMC estimated 6 free parameters, and the priors for these parameters were:

$$
\begin{aligned}
v_{c} & \sim N(0.3,0.3) \\
t_{e r} & \sim T N(0.3,0.3,0, \infty) \\
a & \sim T N(0.2,0.2,0, \infty) \\
\zeta_{c o m p} & \sim T N(0.2,0.2,0, \infty) \\
\zeta_{\text {incomp }} & \sim T N(0.2,0.2,0, \infty) \\
\tau & \sim T N(0.3,0.3,0, \infty)
\end{aligned}
$$


Note that we used fairly constraining priors for the parameters of the automatic activation

- based on the previous ranges of values suggested by Ulrich et al. (2015) and White et al. (2018) - as previous work has displayed "sloppiness" in the estimates of these parameters, where uninformative priors can lead to the estimation of extreme values that seem theoretically unrealistic (Evans \& Servant, 2020). Our parameter recovery used two methods for assessing how well the estimated values matched the generated values across the data sets for each parameter: a qualitative assessment via a scatterplot, and a quantitative assessment via Pearson's correlation coefficient.

We performed four recovery studies, which each fit an identical model (i.e., both in parameterisation and priors), though used a slightly different data generating process. Note that all data sets were generated using simulation via the same method described above, though with a step-size of 0.001 to ensure that (1) our simulated data sets had the same level of precision (i.e., millisecond) as most empirical data sets, and (2) to ensure that our use of a larger step-size (0.01) for fitting did not result in poor recovery when the data contained a higher level of precision. The range of the hypercube for each parameter in the first simulation study was:

$$
\begin{aligned}
v_{c} & \in[0.01,0.3] \\
a & \in[0.05,0.2] \\
\zeta_{\text {comp }} & \in[0,0.1] \\
\zeta_{\text {incomp }} & \in[0,0.1]
\end{aligned}
$$

with the generating values of $\tau$ and $t_{e r}$ fixed to 0.15 and 0.3 , respectively. We chose to fix these parameters in the data generating process (1) to reduce the dimensionality of the 
hypercube for the initial recovery and (2) as these parameters are not of theoretical interest in the current study. Each data set consisted of 1000 trials per condition (i.e., 2000 total trials across the compatible and incompatible conditions). The second, third, and fourth recoveries extended the range of generating parameter values:

$$
\begin{aligned}
v_{c} & \in[0.01,0.6] \\
t_{e r} & \in[0.1,0.5] \\
a & \in[0.01,0.4] \\
\zeta_{\text {comp }} & \in[0,0.2] \\
\zeta_{\text {incomp }} & \in[0,0.2] \\
\tau & \in[0.05,0.55]
\end{aligned}
$$

These recoveries only differed in the number of trials per condition in each data set, in order to assess whether the practical measurement properties of the model deteriorated with decreasing sample size. Specifically, the second, third, and fourth recoveries used 200 (approximately the number of trials per condition in the smallest empirical data set that we assessed), 600, and 1000 trials per condition, respectively.

The parameter recovery assessments for each parameter in each simulation study can be seen in Figure 3. Each plot displays the generated values on the $\mathrm{x}$-axis and the estimated posterior mean parameter values on the $\mathrm{y}$-axis, with the red line $(x=y)$ indicating perfect recovery. The correlation between generated and estimated values is displayed in the topleft hand corner of each plot in red. As shown in the top row, the first simulation study generally display excellent recovery. Note that while we plot the estimated values for $\tau$ and $t_{e r}$ in the first simulation study, we do not interpret them as all data sets used the 
same generating values of these parameters. For the parameters that varied across data sets - including the $\zeta_{c o m p}$ and $\zeta_{\text {incomp }}$ parameters - the estimates from every included data set falls close to the red line, and the correlation between the generated and estimated parameter values are all 0.97 or greater, suggesting excellent recovery. Most importantly, the first simulation study suggests that facilitation and interference effects can be reliably disentangled using our model-based approach.

The extended the range of generating parameter values in the second, third, and fourth recoveries (second, third, and final rows, respectively) appear to make the recovery somewhat poorer, though overall the recovery remains very good in a absolute sense (i.e., high correlations, estimated values in most cases are still very close to the line of perfect recovery). Specifically, these simulation studies display difficulties in accurately recovering the $\tau$ parameter (correlations between 0.85 and 0.86 ), with the range of estimated values increasing as the generating value increases, suggesting that due to a sloppiness in the model, moderate and large values of $\tau$ are difficult to distinguish. In contrast, the estimation of $t_{e r}$ is quite robust, with the estimates from every included data set falling close to the red line, and the correlation between the generated and estimated parameter values being 0.98 or greater. The recovery of $a$ is essentially unchanged from the first simulation study, showing a close qualitative match and 0.98 or greater correlation between the generated and estimated values across each number of trials per condition. However, the recovery of $v_{c}, \zeta_{\text {comp }}$, and $\zeta_{\text {incomp }}$ is slightly worse. Specifically, while the generated and estimated values continue to show a close qualitative match in most instances, there appears to be a tendency for overestimation in a few of the cases with very high generating values, resulting in lower correlations $(0.91-0.96)$. Most importantly, (1) the key parameters of interest $\zeta_{c o m p}$, and $\zeta_{\text {incomp }}$ - still display excellent recovery at an absolute level, again suggesting that facilitation and interference effects can be reliably disentangled using our model-based 
approach, and (2) the recovery appears to be fairly robust across the sample sizes that we assessed, suggesting that facilitation and interference effects can be reliably estimated even in data sets with only 200 trials per condition.

\section{Method}

The previous section of our article proposed an extension of the DMC framework, which intends to provide a model-based approach to disentangling facilitation and interference effects in conflict tasks. Based on our parameter recovery assessments, it appears that our extension can distinguish between facilitation and interference effects in a realistic number of trials, without the need for neutral trials. Therefore, our second aim is to use our extension to estimate facilitation and interference effects in existing conflict task data sets. Specifically, we obtained 9 conflict data sets -6 flanker data sets and 3 Simon data sets - and used our extension to estimate the level of automatic activation in compatible and incompatible trials. This provides a model-based assessment of whether or not facilitation and interference effects (1) are equal, (2) both occur, and (3) are consistent across people, data sets, and tasks. Here, we provide a brief description of each of the 9 data sets - grouped by task - and then our methods of fitting and assessment. Note that the fitting methods are extremely similar to those in the recovery assessment, and therefore, much of this section refers back to the previous section to avoid repetition.

\section{Flanker data sets}

White et al. (2011) Experiment 1. These data consist of 25 participants who were presented with a vertical sequence of five arrows (i.e., $<$ or $>$ ). Participants were instructed to identify whether the central arrow, which was always presented in the centre of the screen, was pointing towards the left or right. Participants were told to perform the task as quickly and accurately as possible, though there was no explicit deadline for responses and 
the stimulus remained on screen until a response was made. After each response there was a 350ms inter-trial interval, with the next stimulus being presented immediately after this interval (i.e., no central fixation cue before the next stimulus). Compatible trials were those where the target and flankers pointed in the same direction, whereas incompatible trials were those where the flankers pointed in the opposite direction to the target. Participants completed 48 practice trials followed by 8 blocks of 96 trials each.

Servant, Montagnini, and Burle (2014) Experiment 1. These data consist of 12 participants who were presented with a horizontal sequence of three coloured circles (i.e., red and blue). Participants were instructed to identify whether the central circle, which was always presented in the centre of the screen, was red or blue. Participants were told to perform the task as quickly and accurately as possible, with an explicit deadline at $1,500 \mathrm{~ms}$ where the trial would terminate. After each response there was a 1,500ms intertrial interval, with the next stimulus being presented immediately after this interval (i.e., no central fixation cue before the next stimulus). Compatible trials were those where the target and flanker circles were the same colours, whereas incompatible trials were those where the flankers supported the opposite response to the target (e.g., a blue target circle flanked by red circles). Participants completed 96 practice trials followed by 24 blocks of 96 trials each. Note that there was also a manipulation of colour intensity of the target, with chroma levels differing between trials with one of six values: $15 \%, 25 \%, 35 \%, 45 \%$, $60 \%, 80 \%$. However, the flankers always had the same colour intensity, which was $80 \%$. As only the target differed in colour intensity based on this manipulation, we assumed that the manipulation selectively influenced the controlled process, and therefore, only influenced $v_{c}$.

Servant et al. (2015). These data consist of 12 participants who were presented with a horizontal sequence of five letters (i.e., 'H' and 'S'). Participants were instructed to 
identify whether the central letter, which was always presented in the centre of the screen, was a 'H' or an 'S'. Participants were told to perform the task as quickly and accurately as possible, though there was no explicit deadline for responses and the stimulus remained on screen until a response was made. After each response there was a 1,000ms inter-trial interval, with the next stimulus being presented immediately after this interval (i.e., no central fixation cue before the next stimulus). Compatible trials were those where the target and flankers were the same letter, whereas incompatible trials were those where the flankers supported the opposite response to the target (e.g., an 'S' target flanked by 'H'). Participants completed 96 practice trials followed by 15 blocks of 96 trials each.

Ulrich et al. (2015). These data consist of 18 participants (though 2 participants were excluded for high error rates; $>10 \%$ ) who were presented with a horizontal sequence of five letters (i.e., 'H' and 'S'). Participants were instructed to identify whether the central letter, which was always presented in the centre of the screen, was a 'H' or an 'S'. Participants were told to perform the task as quickly and accurately as possible, with the stimulus removed after $150 \mathrm{~ms}$ and an explicit deadline at $1,500 \mathrm{~ms}$ where the trial would terminate. After each response there was a 1,000ms inter-trial interval, where participants were provided with feedback about whether they were correct/incorrect, missed, or made an anticipatory response $(<150 \mathrm{~ms})$ with the next trial beginning with fixation cross presented in the center of the screen for $500 \mathrm{~ms}$. Compatible trials were those where the target and flankers were the same letter, whereas incompatible trials were those where the flankers supported the opposite response to the target (e.g., an 'S' target flanked by 'H'). Participants completed 56 practice trials followed by 6 blocks of 56 trials each. Participants in this experiment also completed a Simon task, which was presented in alternating blocks with the flanker task, which we analyse as one of our Simon data sets. 
Servant and Logan (2019) Experiment 1. These data consist of 24 participants who were presented with a horizontal sequence of five letters (i.e., 'H' and 'S'). Participants were instructed to identify whether the central letter, which was always presented in the centre of the screen, was a 'H' or an 'S'. Participants were told to perform the task as quickly and accurately as possible, with an explicit deadline at 1,500ms where the trial would terminate. If participants failed to respond before this deadline, then they were given explicit feedback telling them to respond faster for 5,000ms. After each response there was a $1,500 \mathrm{~ms}$ intertrial interval, with a warning signal being presented before the presentation of the stimulus. Compatible trials were those where the target and flankers were the same letter, whereas incompatible trials were those where the flankers supported the opposite response to the target (e.g., an 'S' target flanked by 'H'). Participants completed 10 practice trials followed by 10 blocks of 100 trials each. It should also be noted that participants were presented with the digit ' 7 ' after their response on half of the trials, with the digit being presented at the location of either the target or one of the flankers. Participants were instructed to press the space bar with their dominant thumb when the digit was present. However, we do not discuss this secondary task further as (1) it is not of interest in the current study, and (2) Experiment 3 in Servant and Logan (2019) suggested that the secondary task did not modulate the magnitude of the flanker effect.

Servant and Logan (2019) Experiment 2. These data consist of 24 participants who were presented with a horizontal sequence of five letters (i.e., 'H' and 'S'). Participants were instructed to identify whether the central letter, which was always presented in the centre of the screen, was a 'H' or an 'S'. Participants were told to perform the task as quickly and accurately as possible, with an explicit deadline at 1,700ms where the trial would terminate. If participants failed to respond before this deadline, then they were given explicit feedback telling them to respond faster for 5,000ms. After each response there was a 1,700ms inter- 
trial interval, with a warning signal being presented before the presentation of the stimulus. Compatible trials were those where the target and flankers were the same letter, whereas incompatible trials were those where the flankers supported the opposite response to the target (e.g., an 'S' target flanked by 'H'). Participants completed 10 practice trials followed by 8 blocks of 100 trials each.

Simon data sets

Pratte, Rouder, Morey, and Feng (2010) Experiment 1. These data consist of 38 participants who were presented with a coloured square (i.e., red and green). Participants were instructed to identify whether the square, which was always presented to either the left or right of the screen, was red or green. Participants were told to perform the task as quickly and accurately as possible, though there was no explicit deadline for responses and the stimulus remained on screen until a response was made. After each response there was a 700ms inter-trial interval, with the next trial beginning with fixation cross presented in the center of the screen for $700 \mathrm{~ms}$. Compatible trials were those where the stimulus and response location matched, whereas incompatible trials were those where they did not. Participants completed 7 blocks of 72 trials each. Participants in this experiment also completed a Stroop task, which was presented after the Simon task, though we do not assess these Stroop data as the focus of the current paper is on the flanker and Simon tasks.

Servant et al. (2014) Experiment 2. These data consist of 12 participants who were presented with a coloured circle (i.e., red and blue). Participants were instructed to identify whether the circle, which was always presented to either the left or right of the screen, was red or blue. Participants were told to perform the task as quickly and accurately as possible, with an explicit deadline at $1,000 \mathrm{~ms}$ where the trial would terminate. After each 
response there was a $1,500 \mathrm{~ms}$ inter-trial interval, with the next stimulus being presented immediately after this interval (i.e., no central fixation cue before the next stimulus). Compatible trials were those where the stimulus and response location matched, whereas incompatible trials were those where they did not. Participants completed 96 practice trials followed by 28 blocks of 96 trials each. Note that there was also a manipulation of colour intensity of the stimulus, with chroma levels differing between trials with one of six values: $15 \%, 25 \%, 35 \%, 45 \%, 60 \%, 80 \%$. As only the task-relevant information differed based on this manipulation, we assumed that the manipulation selectively influenced the controlled process, and therefore, only influenced $v_{c}$.

Ulrich et al. (2015). These data consist of 18 participants (though 2 participants were excluded for high error rates; $>10 \%$ ) who were presented with a letters (i.e., 'H' and 'S'). Participants were instructed to identify whether the letter, which was always presented to either the left or right from a central fixation cross, was a 'H' or an 'S'. Participants were told to perform the task as quickly and accurately as possible, with the stimulus removed after $150 \mathrm{~ms}$ and an explicit deadline at $1,500 \mathrm{~ms}$ where the trial would terminate. After each response there was a 1,000ms inter-trial interval, where participants were provided with feedback about whether they were correct/incorrect, missed, or made an anticipatory response $(<150 \mathrm{~ms})$ with the next trial beginning with fixation cross presented in the center of the screen for $500 \mathrm{~ms}$. Compatible trials were those where the stimulus and response location matched, whereas incompatible trials were those where they did not. Participants completed 56 practice trials followed by 6 blocks of 56 trials each. Participants in this experiment also completed a flanker task, which was presented in alternating blocks with the Simon task, which we analyse as one of our flanker data sets. 


\section{Fitting and assessment}

The methods for fitting the empirical data sets were identical to those in the parameter recovery assessment. In order to assess the consistency of inferences across participants within each study, we performed all assessments at an individual level, meaning that the data of each participant was fit separately. Although there are several advantages to the use of hierarchical models, which constrain the data of all participants to follow a group level distribution, assessments using hierarchical models can often ignore individual differences, and even result in the conclusions of a study being skewed in favour of a few outlying participants (Evans, Hawkins, \& Brown, 2019; though see also Haaf \& Rouder, 2017; Stephan, Penny, Daunizeau, Moran, \& Friston, 2009; Rigoux, Stephan, Friston, \& Daunizeau, 2014).

As our aim was only to assess facilitation and interference effects, our assessment focused on the $\zeta_{c o m p}$ and $\zeta_{\text {incomp }}$ parameters. Specifically, we included two types of assessment of the estimated posterior distributions: a qualitative assessment and a quantitative assessment. The qualitative assessment involved visually inspecting the estimated posterior distributions for each parameter, and whether they differed from (1) one another, which indicates whether or not facilitation and interference effects are equal, and (2) from zero, which indicates whether or not each effect occurs. The quantitative assessment involved calculating the evidence in favour of whether facilitation and/or interference effects occur through the Savage-Dickey ratio approximation to the Bayes factor, as Bayes factors cannot be directly computed for complex cognitive models (Annis, Evans, Miller, \& Palmeri, 2019; Evans \& Annis, 2019; Evans \& Servant, 2020). Note that the Savage-Dickey ratio provides an approximation to the Bayes factor between a point-null hypothesis and an unconstrained alternative hypothesis. In this case, the null hypothesis for the Savage-Dickey ratio assessment of the $\zeta_{c o m p}$ and $\zeta_{\text {incomp }}$ parameters is that only interference and only 
facilitation effects occur, respectively. Therefore, cases where both the $\zeta_{c o m p}$ and $\zeta_{\text {incomp }}$ parameters show evidence for the null hypothesis does not necessarily indicate a lack of either effect, and could instead suggest that either facilitation or interference alone are sufficient to describe the data, and it is unclear which effect is actually present. We interpreted the log-Bayes factor, meaning that the sign of the value indicates whether the evidence is for the null (i.e., negative values) or for the effect (i.e., positive values), and the absolute magnitude indicates the amount of evidence. As in Evans and Servant (2020), we interpreted the strength of evidence according to the guidelines of Jeffreys (1961), where absolute log Bayes factors from 0 to 1.1 indicating weak evidence, 1.2 to 2.2 indicating moderate evidence, 2.3 to 4.6 indicating strong evidence, and 4.7 or greater indicating decisive evidence. Note that for readability, we list any log-Bayes factor above 10 as "> 10".

\section{Results}

Here, we provide the result of our assessments. We first discuss the results of the 6 flanker data sets, and then the 3 Simon data sets. Within each section, we discuss the results for each data set separately, beginning with the qualitative patterns in the posterior distributions for each participants, and using the quantitative measure of evidence in the log-Bayes factor to support our inferences. At the end of each section (i.e., flanker and Simon), we provide a brief summary of the results across all of the data sets within that section. Also note that the goodness-of-fit of our DMC extension to the data (both individual participants and group-averaged quantiles) can be seen in Figure 4, though we do not discuss this in detail as the fit of the model appears adequate for all data sets except Pratte et al. (2010), which we discuss when interpreting the results of this data set. 


\section{Flanker data sets}

The results for the White et al. (2011) data set can be seen in Figure 5. For every participant except one (participant 2), the posterior mean for the $\zeta_{\text {incomp }}$ parameter are higher than for the $\zeta_{c o m p}$ parameter. Furthermore, in almost every case the posterior distribution for the $\zeta_{\text {incomp }}$ parameter is estimated higher than the $\zeta_{\text {comp }}$ parameter with little-to-no overlap between the distributions, indicating that the interference effects in these data appear to be much stronger than the facilitation effects. Moreover, in most cases the $\zeta_{c o m p}$ parameter is estimated very close to zero, and in many of these cases the density of the posterior distribution is peaked around zero, suggesting evidence for an absence of facilitation. In contrast, the $\zeta_{\text {incomp }}$ parameter is mostly estimated away from zero, with very little density close to zero, suggesting clear evidence for the presence of interference. The Bayes factors further support these inferences, with 22 participants showing evidence for the absence of facilitation - 5 weak, 2 moderate, 11 strong, and 4 decisive - and all 25 participants showing evidence for the presence of interference - 1 weak, 1 moderate, 1 strong, and 22 decisive. Therefore, the White et al. (2011) data set appears to suggest the existence of interference effects and the absence of facilitation effects.

The results for the Servant et al. (2014) flanker data set can be seen in Figure 6 . For every participant, the posterior mean for the $\zeta_{\text {incomp }}$ parameter was higher than for the $\zeta_{\text {comp }}$ parameter, and the posterior distribution for the $\zeta_{\text {incomp }}$ parameter is estimated higher than the $\zeta_{\text {comp }}$ parameter with little-to-no overlap between the distributions. This indicates that the interference effects in these data appear to be much stronger than the facilitation effects. Moreover, in every case the $\zeta_{\text {comp }}$ parameter is estimated very close to zero and the density of the posterior distribution is peaked around zero, suggesting

evidence for an absence of facilitation. In contrast, in every case the $\zeta_{\text {incomp }}$ parameter is estimated away from zero, with very little density close to zero, suggesting clear evidence 
for the presence of interference. The Bayes factors further support these inferences, with all 12 participants showing evidence for the absence facilitation - 3 strong and 9 decisive - and all 12 participants showing evidence for the presence of interference - 1 weak and 11 decisive. Therefore, the Servant et al. (2014) flanker data set appears to suggest the existence of interference effects and the absence of facilitation effects.

The results for the Servant et al. (2015) data set can be seen in Figure 7. For every participant, the posterior mean for the $\zeta_{\text {incomp }}$ parameter was higher than for the $\zeta_{\text {comp }}$ parameter. Furthermore, in most case the posterior distribution for the $\zeta_{\text {incomp }}$ parameter is estimated higher than the $\zeta_{\text {comp }}$ parameter with little-to-no overlap between the distributions (though see participants 4,6 , and 8 ). This indicates that the interference effects in these data appear to be much stronger than the facilitation effects, though the trend is somewhat less consistent across participants than the previous data sets. Moreover, in every case the $\zeta_{\text {comp }}$ parameter is estimated very close to zero and the density of the posterior distribution is peaked around zero, suggesting evidence for an absence of facilitation. In contrast, in most cases the $\zeta_{\text {incomp }}$ parameter is estimated away from zero, with very little density close to zero (though again see participants 4, 6, and 8), suggesting clear evidence for the presence of interference. The Bayes factors further support these inferences, with all 12 participants showing evidence for the absence of facilitation - 1 moderate, 6 strong, and 5 decisive - and 11 participants showing evidence for the presence of interference -2 weak and 9 decisive. Therefore, the Servant et al. (2015) data set appears to suggest the existence of interference effects and the absence of facilitation effects.

The results for the Ulrich et al. (2015) flanker data set can be seen in Figure 8. For every participant except two (participant 8 and 16), the posterior mean for the $\zeta_{\text {incomp }}$ parameter was higher than for the $\zeta_{\text {comp }}$ parameter. Furthermore, in the slight majority of cases the posterior distribution for the $\zeta_{\text {incomp }}$ parameter is estimated higher than the $\zeta_{c o m p}$ 
parameter with little-to-no overlap between the distributions (though see participants 2, 4, $8,13,14$, and 16), indicating that the interference effects in these data generally appear to be much stronger than the facilitation effects. This indicates that the interference effects in these data appear to be much stronger than the facilitation effects, though again, the trend is somewhat less consistent across participants than the previous data sets. Moreover, in every case the $\zeta_{c o m p}$ parameter is estimated very close to zero, and in most cases the density of the posterior distribution is peaked around zero, suggesting evidence for an absence of facilitation. In contrast, in the majority of cases the $\zeta_{\text {incomp }}$ parameter is estimated away from zero, with very little density close to zero (though again see participants $2,4,8,13$, 14, and 16), suggesting clear evidence for the presence of interference. The Bayes factors further support these inferences, with all 16 participants showing evidence for the absence facilitation - 2 weak, 2 moderate, 6 strong, and 6 decisive - and 10 participants showing evidence for the presence of interference - 1 weak, 1 moderate, and 8 decisive. Therefore, the Ulrich et al. (2015) flanker data set appears to suggest the existence of interference effects and the absence of facilitation effects.

The results for the first Servant and Logan (2019) data set can be seen in Figure 9. For the majority of participants (15 of 24$)$, the posterior mean for the $\zeta_{\text {incomp }}$ parameter was higher than for the $\zeta_{\text {comp }}$ parameter, suggesting more ambiguity across participants in this data set than the previous data sets. Furthermore, the distributional ordering was also much less clear in this data set, with the majority of participants having largely overlapping distributions. However, of the 10 participants that appear to have largely separated distributions (participants 1, 2, 3, 4, 14, 15, 17, 22, 23, 24), only one showed higher estimates of the $\zeta_{\text {comp }}$ parameter than the $\zeta_{\text {incomp }}$ parameter (participant 17). Furthermore, of the participants that showed some slight separation in their distributions (participants $5,6,7,9,11,12,13,19,20)$, only one showed higher estimates of the $\zeta_{\text {comp }}$ parameter 
than the $\zeta_{\text {incomp }}$ parameter (participant 6 ). Together, this seems to indicate - albeit to a limited degree - that the interference effects in these data appear to be stronger than the facilitation effects, though again, the trend is somewhat less consistent across participants than the previous data sets. Moreover, in most cases the $\zeta_{c o m p}$ parameter is estimated very close to zero and the density of the posterior distribution is peaked around zero, suggesting evidence for an absence of facilitation. In contrast, in most cases the $\zeta_{\text {incomp }}$ parameter is estimated away from zero, and in the majority of cases there is little density close to zero, suggesting some - again, albeit limited - evidence for the presence of interference. The Bayes factors further support these inferences, with 21 participants showing evidence for the absence facilitation - 1 weak, 7 moderate, and 13 strong - and 12 participants showing evidence for the presence of interference - 3 weak, 1 moderate, 3 strong, and 5 decisive. Therefore, the first Servant and Logan (2019) data set appears to suggest the absence of facilitation effects and - to some extent - the existence of interference effects.

The results for the second Servant and Logan (2019) data set can be seen in Figure 10. For the majority of participants (16 of 24$)$, the posterior mean for the $\zeta_{\text {incomp }}$ parameter was higher than for the $\zeta_{\text {comp }}$ parameter, suggesting more ambiguity across participants in this data set than the previous data sets (apart from the first Servant \& Logan, 2019 data set, where the ambiguity is similar). Furthermore, the distributional ordering was also much less clear in this data set, with the majority of participants having largely overlapping distributions. However, of the 13 participants that appeared to have largely separated distributions (participants $2,4,7,8,10,11,12,14,17,18,20,22,23$ ), only two showed higher estimates of the $\zeta_{\text {comp }}$ parameter than the $\zeta_{\text {incomp }}$ parameter (participants 10 and 17). Furthermore, all of the participants that showed some slight separation in their distributions (participants 5, 15, 16, 21, 24) showed higher estimates of the $\zeta_{\text {incomp }}$ parameter than the $\zeta_{\text {comp }}$ parameter. Together, this seems to indicate - albeit to a limited 
degree - that the interference effects in these data appear to be stronger than the facilitation effects, though again, the trend is somewhat less consistent across participants than the first four data sets, and more similar to the first Servant and Logan (2019) data set. Moreover, in most cases the $\zeta_{c o m p}$ parameter is estimated very close to zero and the density of the posterior distribution is peaked around zero, suggesting evidence for an absence of facilitation. In contrast, in the majority of cases the $\zeta_{\text {incomp }}$ parameter is estimated away from zero and there is little density close to zero, suggesting some - again, albeit limited - evidence for the presence of interference. The Bayes factors further support these inferences, with 21 participants showing evidence for the absence of facilitation -4 weak, 3 moderate, 12 strong, and 2 decisive - and 11 participants showing evidence for the presence of interference - 1 weak, 3 moderate, 1 strong, and 6 decisive. However, it should be noted that one participant did show decisive evidence for the presence of facilitation (participant 17), suggesting that facilitation effects may be present in some rare cases. Therefore, the second Servant and Logan (2019) data set appears to suggest the absence of facilitation effects, and - to some extent - the existence of interference effects.

Overall, the 6 flanker data sets appear to show fairly consistent results. Four data sets - White et al. (2011), Servant et al. (2014), Servant et al. (2015), and Ulrich et al. (2015) displayed fairly clear evidence for the presence of interference and the absence of facilitation, though it should be noted that these effects were rarely consistent across every participants, and were mostly only consistent across the vast majority of participants. The two data sets from Servant and Logan (2019) also displayed fairly clear evidence for the absence of facilitation, though showed lesser evidence for the presence of interference. Although these overall results were qualitatively similar to the previous four studies, the results regarding the presence of interference were less consistent across participants, suggesting some ambiguity in the results. However, the overall trend across these flanker data sets 
appears to show a presence of interference and an absence of facilitation, suggesting that interference effects occur within the flanker task, though facilitation effects do not.

Simon data sets

The results for the Pratte et al. (2010) data set can be seen in Figure 11. In general, there does not appear to be any clear patterns across participants within this data set. For just over half of the participants (22 of 38) the posterior mean for the $\zeta_{\text {incomp }}$ parameter was higher than for the $\zeta_{c o m p}$ parameter, though clear distribution separation appears rare. Furthermore, in many cases where there is clear distribution separation, the effect is in the opposite direction, where the posterior distribution for the $\zeta_{c o m p}$ parameter is estimated higher than the $\zeta_{\text {incomp }}$ parameter (participants 14, 17, 21, 34, 37, 38). This seems to suggest that there is no clear difference between facilitation and interference effects in these data. Moreover, most participants have one of the two parameters estimated very close to zero with the density of the posterior distribution peaked around zero, though it is inconsistent between participants whether this is the $\zeta_{\text {comp }}$ parameter or the $\zeta_{\text {incomp }}$ parameter. The Bayes factors further support this ambiguity, with 34 participants showing evidence for the absence of facilitation - 3 weak, 12 moderate, and 19 strong - and 37 participants showing evidence for the absence of interference - 3 weak, 11 moderate, 20 strong, and 3 decisive. Therefore, the Pratte et al. (2010) data set appears to show no clear evidence to distinguish between facilitation and interference effects. One potential reason for these ambiguous results could be that the DMC model is not appropriate for this data set. While the design of Pratte et al. (2010) appears similar to the other Simon data sets in our study, the empirical quantiles (see Figure 4) are substantially more variable than the other Simon data sets, extending out to $1,300 \mathrm{~ms}$ for some participants. Furthermore, the fit of our DMC extension is much poorer to the data of Pratte et al. (2010) than any of the other data sets within our study, with the model even showing misfit to the group-averaged quantiles. 
Therefore, the ambiguity of these results may be more reflective of model inadequacy for this data set, rather than difficulties distinguishing between facilitation and interference effects in the Simon task.

The results for the Servant et al. (2014) Simon data set can be seen in Figure 12. For every participant, the posterior mean for the $\zeta_{\text {incomp }}$ parameter was higher than for the $\zeta_{\text {comp }}$ parameter, and the posterior distribution for the $\zeta_{\text {incomp }}$ parameter is estimated higher than the $\zeta_{c o m p}$ parameter with little-to-no overlap between the distributions. This indicates that the interference effects in these data appear to be much stronger than the facilitation effects. Moreover, in every case the $\zeta_{\text {comp }}$ parameter is estimated very close to zero and the density of the posterior distribution is peaked around zero, suggesting evidence for an absence of facilitation. In contrast, in every case the $\zeta_{\text {incomp }}$ parameter is estimated away from zero, with very little density close to zero, suggesting clear evidence for the presence of interference. The Bayes factors further support these inferences, with all 12 participants showing evidence for the absence of facilitation -2 strong and 10 decisive - and 11 participants showing evidence for the presence of interference - all decisive. Therefore, the Servant et al. (2014) Simon data set appears to suggest the existence of interference effects and the absence of facilitation effects.

The results for the Ulrich et al. (2015) Simon data set can be seen in Figure 13. For every participant, the posterior mean for the $\zeta_{\text {incomp }}$ parameter was higher than for the $\zeta_{\text {comp }}$ parameter, and the posterior distribution for the $\zeta_{\text {incomp }}$ parameter is estimated higher than the $\zeta_{\text {comp }}$ parameter with little-to-no overlap between the distributions (though see participants 6 and 16). This indicates that the interference effects in these data appear to be much stronger than the facilitation effects. Moreover, in every case the $\zeta_{c o m p}$ parameter is estimated very close to zero and the density of the posterior distribution is peaked around zero, suggesting evidence for an absence of facilitation. In contrast, in most 
cases the $\zeta_{\text {incomp }}$ parameter is estimated away from zero, with very little density close to zero (though again see participants 6 and 16), suggesting clear evidence for the presence of interference. The Bayes factors further support these inferences, with all 16 participants showing evidence for the absence facilitation - 4 strong and 12 decisive - and 13 participants showing evidence for the presence of interference - 1 moderate and 12 decisive. Therefore, the Ulrich et al. (2015) Simon data set appears to suggest the existence of interference effects and the absence of facilitation effects.

Overall, the 3 Simon data sets appeared to show somewhat consist results. Two data sets - Servant et al. (2014) and Ulrich et al. (2015) - displayed very clear evidence for the presence of interference and the absence of facilitation. In contrast, the Pratte et al. (2010) data set displayed no clear evidence in either direction, though these ambiguous results could be potentially explained by model inadequacy, as suggested by the poorer goodness-of-fit to this data set. Consequently, the overall trend across these Simon data sets appears to suggest a presence of interference and an absence of facilitation, suggesting that interference effects occur within the Simon task, though facilitation effects do not.

\section{Discussion}

The current study aimed to develop a model-based approach to disentangling facilitation and inference effects in conflict tasks. Previous studies have attempted to separate facilitation and interference effects using neutral trials; an experimental-based approach that involves contrasting trials without task-irrelevant information to compatible and incompatible trials. However, these types of cost-benefits analyses rely on the logic of Donder's subtractive method (Donders, 1969) - that neutral trials do not provide any form of cue towards either response alternative, and that processing only differs along this dimension compared to compatible and incompatible trials. Model-based approaches provide an 
alternative to experimental-based approaches, replacing the assumptions about the experimental conditions with explicit assumptions contained within the model based on psychological theory (Crüwell et al., 2019). However, no model-based approaches currently exist for separating facilitation and interference effects in conflict tasks. Our study extended the DMC framework (Ulrich et al., 2015) to separate facilitation and interference effects by allowing the maximum amplitude of the time-varying automatic activation to vary between compatible and incompatible trials. Furthermore, our comprehensive parameter recovery assessment displayed the robust properties of our DMC extension, where facilitation and interference effects could be reliably estimated with as few as 200 trials per condition.

Based on the robust measurement properties of our DMC extension, the current study also aimed to implement our novel model-based approach to disentangle facilitation and interference effects in 9 different data sets from two paradigmatic conflict tasks - the flanker task (6) and the Simon task (3) - and importantly, assess whether there was evidence for both effects in both tasks. We provided separate assessments for each individual participant, in order to assess the consistency of inferences across participants as well as across studies, as these potential ambiguities can often be ignored by common hierarchical methods (Evans, Hawkins, \& Brown, 2019; though see also Haaf \& Rouder, 2017; Stephan et al., 2009; Rigoux et al., 2014). The results across the 6 flanker data sets showed fairly clear evidence for the presence of interference and the absence of facilitation, particularly in the data sets of White et al. (2011), Servant et al. (2014), Servant et al. (2015), Ulrich et al. (2015). However, it should also be noted that there was some variability between individuals - which could either reflect noise or meaningful individual differences in ability or strategy (Haaf \& Rouder, 2017) - and that these trends were merely those of the vast majority of participants. The results across 2 of the 3 Simon data sets showed very clear evidence for the presence of interference and the absence of facilitation, though the Pratte 
et al. (2010) data set showed extremely ambiguous results, which may have been the result of model inadequacy. While the strong trends in the Servant et al. (2014) and Ulrich et al. (2015) data sets appear to indicate some evidence for the presence of interference and the absence of facilitation in the Simon task, further research is needed to more clearly establish this finding due to the limited number of Simon data sets assessed in the current study. Overall, the data sets assessed within this paper appear to show clear model-based evidence for conflict effects being driven by interference effects, with facilitation effects being a minor part of conflict effects, if they are present at all.

However, we wish to note that our study does not intend to claim that our modelbased approach is superior to experimental-based approaches for distinguishing between facilitation and interference effects, such as neutral trials. Rather, we believe that modelbased and experimental-based approaches have unique strengths and weaknesses, and should be used to complement one another. A strength of model-based approaches, such as the DMC extension that we propose here, is the ability to decompose data into direct measurements of the underlying cognitive process using the assumptions of theory. However, a weakness of model-based approaches is that they rely on the assumptions of theory, and that if the assumptions of the theory are invalid, then the inferences made from the approach may also be invalid. In contrast, a strength of experimental-based approaches is that they do not directly rely on theory, meaning that one can make conclusions about certain parts of the process while remaining agnostic to other parts of the process. However, a weakness of experimental-based approaches is that they rely on the validity of the experimental manipulation, in that it influences, and only influences, the part of the cognitive process that the experimenter believes it should influence (i.e., the selective influence assumption; see Dutilh et al., 2019; Evans, 2020b for recent discussions of the issues with this assumption in other areas). Importantly, as discussed by Jonides and Mack (1984), 
cost-benefit analyses - such as neutral trials - strongly rely on the assumptions of Donder's subtractive method (Donders, 1969), and violations of these assumptions - which our literature review in the introduction suggests is plausible for neutral trials in conflict tasks - could render the inferences of these experimental-based approaches invalid. We believe that our model-based approach brings an important, novel perspective to the debate on facilitation and interference effects, which should be used in combination with results from neutral trials, rather than in place of them. Overall, we believe that researchers should look for converging sources of evidence from both experimental and model-based approaches similar to many other areas of cognitive psychology - and that our approach provides a robust model-based framework to pair with existing experimental-based approaches. Furthermore, we do not intend to claim that our study provides conclusive evidence for a lack of facilitation effects in the flanker and/or Simon task. A great deal of future research is needed to both (1) further establish these findings within our model-based framework, and (2) resolve any discrepancies between these findings and those from neutral trials.

One interesting aspect of our findings are the consistent results between the flanker and Simon tasks, where both tasks appear to show evidence for the presence of interference and the absence of facilitation. A longstanding debate within the literature has been whether all conflict tasks probe the same underlying construct. Previous research has highlighted several differences in the behavioural trends observed in the flanker and Simon tasks, with the flanker effect increasing over time (i.e., a positive delta plot; Pratte et al., 2010) and under higher working memory load (Lavie, Hirst, de Fockert, \& Viding, 2004), and the Simon effect decreasing over time (i.e., a negative delta plot; Pratte et al., 2010) and under higher working memory load (Wühr \& Biebl, 2011). However, these differences between tasks can be largely accommodated by common theoretical frameworks that allow for differences in the relative time course of location-based and flanker-based 
automatic activation, such as DMC (Ulrich et al., 2015). A more recent study investigating individual differences in conflict tasks found low correlations between the measured conflict effects across tasks, suggesting that these tasks may probe different underlying constructs (Rey-Mermet \& Gade, 2018). However, as pointed out by Rouder, Kumar, and Haaf (2019), correlations between conflict effects can be spurious with an insufficient number of trials per person, meaning that these low correlations may reflect an inadequate experimental design, rather than a difference between tasks. We believe that the consistency of our findings between tasks suggest either (1) a common underlying construct, or (2) different underlying constructs that manifest in a similar manner, though we do not believe that our findings provide much distinction between these possibilities. However, when viewing our findings in combination with those of Ulrich et al. (2015) and Rouder et al. (2019), we believe that previous claims of different conflict tasks having different underlying constructs begin to look questionable. Importantly, we believe that our model-based approach to estimating facilitation and interference effects, combined with recent joint modelling approaches to estimating correlations within hierarchical models (Turner, Forstmann, et al., 2013; Turner, Rodriguez, Norcia, McClure, \& Steyvers, 2016; Evans, Steyvers, \& Brown, 2018; Turner, Van Maanen, \& Forstmann, 2015; Knowles, Evans, \& Burke, 2019; Turner, Wang, \& Merkle, 2017), could provide a useful tool for future research assessing the relationship between conflict tasks.

Our successful extension of the DMC framework also provides motivation for further comparisons between the conflict diffusion models that include our DMC extension. Specifically, the current study consistently found an asymmetry between facilitation and interference effects, suggesting that our DMC extension provides the best account of the data when facilitation and interference effects are not equal. In contrast, the standard DMC framework assumes that facilitation and interference effects are equal, meaning that 
this constraint likely results in a poorer account of the data than our DMC extension. Importantly, our previous work suggested that the standard DMC systematically provides a poorer account of flanker data than the SSP and DSTP, suggesting that the SSP and DSTP may provide a more accurate account of the process underlying the flanker task (Evans \& Servant, 2020; though see Servant \& Evans, 2020 for a DMC account of a flanker spacing manipulation). However, it seems plausible that our DMC extension would provide a better account of empirical data, and therefore, may result in DMC providing an equally good account of flanker data as the SSP and DSTP. However, it should also be noted that previous studies assessing the mimicry between the conflict diffusion models do not include our DMC extension (e.g., White et al., 2018), and therefore, it is possible that our extension will more closely mimic other models, such as the SSP as both models can predict an absence of facilitation. Therefore, future research should aim to both (1) assess the mimicry between our DMC extension and the other conflict diffusion models, and (2) include our DMC extension in comparisons between conflict diffusion models regarding which best accounts for empirical data. 


\section{References}

Annis, J., Evans, N. J., Miller, B. J., \& Palmeri, T. J. (2019). Thermodynamic integration and steppingstone sampling methods for estimating bayes factors: A tutorial. Journal of mathematical psychology, 89, 67-86.

Beller, H. K. (1971). Priming: Effects of advance information on matching. Journal of Experimental Psychology, 87(2), 176.

Boehm, U., Annis, J., Frank, M. J., Hawkins, G. E., Heathcote, A., Kellen, D., ... Wagenmakers, E.-J. (2018). Estimating across-trial variability parameters of the diffusion decision model: Expert advice and recommendations. Journal of Mathematical Psychology, 87, 46-75.

Brown, S. D., Ratcliff, R., \& Smith, P. L. (2006). Evaluating methods for approximating stochastic differential equations. Journal of Mathematical Psychology, 50, 402-410.

Brown, T. L. (2011, February). The relationship between Stroop interference and facilitation effects: statistical artifacts, baselines, and a reassessment. Journal of Experimental Psychology. Human Perception and Performance, 37(1), 85-99. doi: 10.1037/a0019252

Burle, B., Possamaï, C.-A., Vidal, F., Bonnet, M., \& Hasbroucq, T. (2002, November). Executive control in the Simon effect: an electromyographic and distributional analysis. Psychological Research, 66 (4), 324-336. doi: 10.1007/s00426-002-0105-6

Craft, J. L., \& Simon, J. R. (1970, March). Processing symbolic information from a visual display: interference from an irrelevant directional cue. Journal of Experimental Psychology, 83(3), 415-420. doi: 10.1037/h0028843

Crüwell, S., Stefan, A. M., \& Evans, N. J. (2019, December). Robust Standards in Cognitive Science. Computational Brain \&3 Behavior, 2(3), 255-265. Retrieved 2020-04-23, from https:// doi.org/10.1007/s42113-019-00049-8 doi: 10.1007/s42113-019-00049-8

De Jong, R., Liang, C. C., \& Lauber, E. (1994, August). Conditional and unconditional automaticity: a dual-process model of effects of spatial stimulus-response correspondence. Journal of Experimental Psychology. Human Perception and Performance, 20(4), 731-750. doi: 10.1037//0096-1523.20.4.731

Donders, F. C. (1969). On the speed of mental processes. Acta Psychologica, 30, 412-431. doi: 
10.1016/0001-6918(69)90065-1

Dutilh, G., Annis, J., Brown, S. D., Cassey, P., Evans, N. J., Grasman, R. P. P. P., .. Donkin, C. (2019, August). The Quality of Response Time Data Inference: A Blinded, Collaborative Assessment of the Validity of Cognitive Models. Psychonomic Bulletin \& Review, 26(4), 10511069. Retrieved 2020-07-21, from https://doi.org/10.3758/s13423-017-1417-2 doi: $10.3758 / \mathrm{s} 13423-017-1417-2$

Eriksen, B. A., \& Eriksen, C. W. (1974, January). Effects of noise letters upon the identification of a target letter in a nonsearch task. Perception 83 Psychophysics, 16(1), 143-149. Retrieved 2019-09-27, from https://doi.org/10.3758/BF03203267 doi: 10.3758/BF03203267

Eriksen, C. W., \& Eriksen, B. A. (1979, May). Target redundancy in visual search: Do repetitions of the target within thedisplay impair processing? Perception \& Psychophysics, 26(3), 195205. Retrieved 2020-05-28, from https://doi.org/10.3758/BF03199869 doi: 10.3758/ BF03199869

Eriksen, C. W., \& St James, J. D. (1986, October). Visual attention within and around the field of focal attention: a zoom lens model. Perception \& Psychophysics, 40(4), 225-240. doi: 10.3758/bf03211502

Evans, N. J. (2019). A method, framework, and tutorial for efficiently simulating models of decision-making. Behavior research methods, 51(5), 2390-2404.

Evans, N. J. (2020a). Same model, different conclusions: An identifiability issue in the linear ballistic accumulator model of decision-making. PsyArXiv.

Evans, N. J. (2020b). Think fast! the implications of emphasizing urgency in decision-making. PsyArXiv.

Evans, N. J., \& Annis, J. (2019). Thermodynamic integration via differential evolution: A method for estimating marginal likelihoods. Behavior research methods, 1-18.

Evans, N. J., Bennett, A. J., \& Brown, S. D. (2019). Optimal or not; depends on the task. Psychonomic Bulletin \& Review, 26(3), 1027-1034.

Evans, N. J., \& Brown, S. D. (2017). People adopt optimal policies in simple decision-making, after practice and guidance. Psychonomic Bulletin \& Review, 24(2), 597-606.

Evans, N. J., Brown, S. D., Mewhort, D. J., \& Heathcote, A. (2018). Refining the law of practice. 
Psychological Review, 125(4), 592.

Evans, N. J., Dutilh, G., Wagenmakers, E.-J., \& van der Maas, H. L. (2020). Double responding: A new constraint for models of speeded decision making. Cognitive Psychology, 121, 101292.

Evans, N. J., Hawkins, G., \& Brown, S. (2019). The role of passing time in decision-making. Journal of Experimental Psychology: Learning, Memory, $\mathcal{G}$ Cognition.

Evans, N. J., \& Servant, M. (2020). A comparison of conflict diffusion models in the flanker task through pseudolikelihood Bayes factors. Psychological Review, 127(1), 114-135. doi: $10.1037 /$ rev0000165

Evans, N. J., Steyvers, M., \& Brown, S. D. (2018). Modeling the covariance structure of complex datasets using cognitive models: An application to individual differences and the heritability of cognitive ability. Cognitive science, 42(6), 1925-1944.

Evans, N. J., Tillman, G., \& Wagenmakers, E.-J. (2020). Systematic and random sources of variability in perceptual decision-making: Comment on Ratcliff, Voskuilen, and McKoon (2018). Psychological Review.

Evans, N. J., Trueblood, J. S., \& Holmes, W. R. (2020). A parameter recovery assessment of time-variant models of decision-making. Behavior research methods, 52(1), 193-206.

Evans, N. J., \& Wagenmakers, E.-J. (2020). Evidence accumulation models: Current limitations and future directions. The Quantitative Methods for Psychology.

Gutenkunst, R. N., Waterfall, J. J., Casey, F. P., Brown, K. S., Myers, C. R., \& Sethna, J. P. (2007). Universally sloppy parameter sensitivities in systems biology models. PLoS Computational Biology, 3(10), e189.

Haaf, J. M., \& Rouder, J. N. (2017). Developing constraint in bayesian mixed models. Psychological Methods, 22(4), 779 .

Hawkins, G. E., Forstmann, B. U., Wagenmakers, E.-J., Ratcliff, R., \& Brown, S. D. (2015). Revisiting the evidence for collapsing boundaries and urgency signals in perceptual decisionmaking. Journal of Neuroscience, 35, 2476-2484.

Hedge, C., Vivian-Griffiths, S., Powell, G., Bompas, A., \& Sumner, P. (2019, October). Slow and steady? Strategic adjustments in response caution are moderately reliable and correlate across tasks. Consciousness and Cognition, 75, 102797. Retrieved 2020-09-23, 
from http://www.sciencedirect.com/science/article/pii/S1053810019300571 doi: 10.1016/j.concog.2019.102797

Holmes, W. R. (2015). A practical guide to the probability density approximation (pda) with improved implementation and error characterization. Journal of Mathematical Psychology, $68,13-24$.

Hommel, B. (1993, October). The relationship between stimulus processing and response selection in the Simon task: Evidence for a temporal overlap. Psychological Research, 55(4), 280290. Retrieved 2020-05-28, from https://doi.org/10.1007/BF00419688 doi: 10.1007/ BF00419688

Hommel, B. (1994). Spontaneous decay of response-code activation. Psychological Research, 56 (4), 261-268. doi: 10.1007/BF00419656

Howard, Z. L., Evans, N. J., Innes, R. J., Brown, S. D., \& Eidels, A. (2020). How is multi-tasking different from increased difficulty? Psychonomic Bulletin 83 Review.

Hübner, R., \& Pelzer, T. (2020, February). Improving parameter recovery for conflict drift-diffusion models. Behavior Research Methods. doi: 10.3758/s13428-020-01366-8

Hübner, R., Steinhauser, M., \& Lehle, C. (2010, July). A dual-stage two-phase model of selective attention. Psychological Review, 117(3), 759-784. doi: 10.1037/a0019471

Hübner, R., \& Töbel, L. (2019, March). Conflict resolution in the Eriksen flanker task: Similarities and differences to the Simon task. PLOS ONE, 14(3), e0214203. Retrieved 202009-23, from https://journals.plos.org/plosone/article?id=10.1371/journal . pone .0214203 (Publisher: Public Library of Science) doi: 10.1371/journal.pone.0214203

Jeffreys, H. (1961). Theory of probability. Oxford, England: Oxford University Press.

Jonides, J., \& Mack, R. (1984). On the cost and benefit of cost and benefit. Psychological Bulletin, 96(1), 29-44. doi: 10.1037/0033-2909.96.1.29

Kahneman, D., \& Tversky, A. (1979). Prospect theory: An analysis of decision under risk. Econometrica, 47, 263-291.

Knowles, J. P., Evans, N. J., \& Burke, D. (2019). Some evidence for an association between early life adversity and decision urgency. Frontiers in psychology, 10, 243.

Kornblum, S., Hasbroucq, T., \& Osman, A. (1990, April). Dimensional overlap: cognitive basis 
for stimulus-response compatibility-a model and taxonomy. Psychological Review, 97(2), 253-270. doi: 10.1037/0033-295x.97.2.253

Lavie, N., Hirst, A., de Fockert, J. W., \& Viding, E. (2004, September). Load theory of selective attention and cognitive control. Journal of Experimental Psychology. General, 133(3), 339354. doi: 10.1037/0096-3445.133.3.339

Lerche, V., Bucher, A., \& Voss, A. (2019). Processing emotional expressions under fear of rejection: Findings from diffusion model analyses. Emotion.

Lerche, V., von Krause, M., Voss, A., Frischkorn, G. T., Schubert, A.-L., \& Hagemann, D. (2020). Diffusion modeling and intelligence: Drift rates show both domaingeneral and domain-specific relations with intelligence. Journal of Experimental Psychology: General.

Lerche, V., \& Voss, A. (2016). Model complexity in diffusion modeling: Benefits of making the model more parsimonious. Frontiers in psychology, 7, 1324.

Lerche, V., Voss, A., \& Nagler, M. (2017). How many trials are required for parameter estimation in diffusion modeling? a comparison of different optimization criteria. Behavior Research Methods, 49(2), 513-537.

Lev, M., Yehezkel, O., \& Polat, U. (2014, February). Uncovering foveal crowding? Scientific Reports, 4, 4067. doi: 10.1038/srep04067

Lin, H., Saunders, B., Friese, M., Evans, N. J., \& Inzlicht, M. (2020). Strong effort manipulations reduce response caution: A preregistered reinvention of the ego-depletion paradigm. Psychological Science.

Mattler, U. (2003, July). Delayed flanker effects on lateralized readiness potentials. Experimental Brain Research, 151(2), 272-288. Retrieved 2020-09-23, from https://doi.org/10.1007/ s00221-003-1486-5 doi: 10.1007/s00221-003-1486-5

McKay, M. D., Beckman, R. J., \& Conover, W. J. (1979). Comparison of three methods for selecting values of input variables in the analysis of output from a computer code. Technometrics, $21(2), 239-245$.

Meyer, D. E., \& Schvaneveldt, R. W. (1971). Facilitation in recognizing pairs of words: Evidence of a dependence between retrieval operations. Journal of Experimental Psychology, 90, 227-234.

Miller, J. (1991, May). The flanker compatibility effect as a function of visual angle, attentional 
focus, visual transients, and perceptual load: A search for boundary conditions. Perception E3 Psychophysics, 49(3), 270-288. Retrieved 2019-12-19, from https://doi.org/10.3758/ BF03214311 doi: 10.3758/BF03214311

Mittelstädt, V., \& Miller, J. (2018, August). Redundancy gain in the Simon Task: Does increasing relevant activation reduce the effect of irrelevant activation? Journal of Experimental Psychology. Human Perception and Performance, 44 (8), 1153-1167. doi: 10.1037/xhp0000523

Osth, A. F., \& Dennis, S. (2015). Sources of interference in item and associative recognition memory. Psychological review, 122(2), 260.

Posner, M., \& Snyder, C. (1975). Attention and cognitive control. in RL Solso, (Ed), Information processing and cognition (pp 55-85) Hillsdale. NJ Erlbaum.

Pratte, M. S., Rouder, J. N., Morey, R. D., \& Feng, C. (2010, October). Exploring the differences in distributional properties between Stroop and Simon effects using delta plots. Attention, Perception, 63 Psychophysics, 72(7), 2013-2025. Retrieved 2019-09-27, from https://doi .org/10.3758/APP.72.7.2013 doi: 10.3758/APP.72.7.2013

Purcell, B. A., Heitz, R. P., Cohen, J. Y., Schall, J. D., Logan, G. D., \& Palmeri, T. J. (2010). Neurally constrained modeling of perceptual decision making. Psychological Review, 117, $1113-1143$.

Ratcliff, R. (1978). A theory of memory retrieval. Psychol. Rev, 85(2), 59-108.

Ratcliff, R., \& Rouder, J. N. (1998). Modeling Response Times for Two-Choice Decisions. Psychological Science, 9(5), 347-356. Retrieved 2019-09-27, from https://www.jstor.org/ stable/40063319

Ratcliff, R., \& Strayer, D. (2014). Modeling simple driving tasks with a one-boundary diffusion model. Psychonomic Bulletin \& Review, 21(3), 577-589.

Ratcliff, R., Thapar, A., \& McKoon, G. (2001, June). The effects of aging on reaction time in a signal detection task. Psychology and Aging, 16(2), 323-341.

Ratcliff, R., \& Tuerlinckx, F. (2002, September). Estimating parameters of the diffusion model: Approaches to dealing with contaminant reaction times and parameter variability. Psychonomic Bulletin \& Review, 9(3), 438-481. Retrieved 2020-04-10, from https://doi.org/ 10.3758/BF03196302 doi: 10.3758/BF03196302 
Rey-Mermet, A., \& Gade, M. (2018, October). Inhibition in aging: What is preserved? What declines? A meta-analysis. Psychonomic Bulletin $\mathscr{E}$ Review, 25(5), 1695-1716. doi: 10.3758/ s13423-017-1384-7

Ridderinkhof, K. R. (2002). Activation and suppression in conflict tasks: empirical clarification through distributional analyses. In Attention and Performance, Vol. XIX, Common Mechanisms in Perception and Action (W. Prinz and B. Hommel ed., pp. 494-519). Oxford: Oxford University Press.

Ridderinkhof, K. R., van der Molen, M. W., \& Bashore, T. R. (1995, November). Limits on the application of additive factors logic: Violations of stage robustness suggest a dual-process architecture to explain flanker effects on target processing. Acta Psychologica, 90(1), 2948. Retrieved 2019-12-12, from http://www.sciencedirect.com/science/article/pii/ 0001691895000310 doi: 10.1016/0001-6918(95)00031-O

Rigoux, L., Stephan, K. E., Friston, K. J., \& Daunizeau, J. (2014). Bayesian model selection for group studies - revisited. Neuroimage, 84, 971-985.

Roitman, J. D., \& Shadlen, M. N. (2002). Responses of neurons in the lateral intraparietal area during a combined visual discrimination reaction time task. Journal of Neuroscience, 22, 9475-9489.

Rouder, J., Kumar, A., \& Haaf, J. M. (2019). Why most studies of individual differences with inhibition tasks are bound to fail. PsyArXiv.

Salthouse, T. A. (1996). The processing-speed theory of adult age differences in cognition. Psychological Review, 103, 403-ñ428.

Servant, M., \& Evans, N. J. (2020, July). A diffusion model analysis of the effects of aging in the Flanker Task. Psychology and Aging. doi: 10.1037/pag0000546

Servant, M., \& Logan, G. D. (2019, June). Dynamics of attentional focusing in the Eriksen flanker task. Attention, Perception \& Psychophysics. doi: 10.3758/s13414-019-01796-3

Servant, M., Montagnini, A., \& Burle, B. (2014, July). Conflict tasks and the diffusion framework: Insight in model constraints based on psychological laws. Cognitive Psychology, 72, 162-195. doi: 10.1016/j.cogpsych.2014.03.002

Servant, M., White, C., Montagnini, A., \& Burle, B. (2015, July). Using Covert Response Activation 
to Test Latent Assumptions of Formal Decision-Making Models in Humans. The Journal of Neuroscience: The Official Journal of the Society for Neuroscience, 35(28), 10371-10385. doi: 10.1523/JNEUROSCI.0078-15.2015

Servant, M., White, C., Montagnini, A., \& Burle, B. (2016, October). Linking Theoretical Decisionmaking Mechanisms in the Simon Task with Electrophysiological Data: A Model-based Neuroscience Study in Humans. Journal of Cognitive Neuroscience, 28(10), 1501-1521. doi: 10.1162/jocn_a_00989

Shiffrin, R. M., \& Steyvers, M. (1997). A model for recognition memory: REM-retrieving effectively from memory. Psychonomic Bulletin \& Review, 4, 145-166.

Simon, J. R., \& Craft, J. L. (1970). Effects of an irrelevant auditory stimulus on visual choice reaction time. Journal of Experimental Psychology, 86(2), 272.

Simon, J. R., \& Small Jr., A. M. (1969). Processing auditory information: Interference from an irrelevant cue. Journal of Applied Psychology, 53(5), 433-435. doi: 10.1037/h0028034

Smith, P. L. (2000). Stochastic dynamic models of response time and accuracy: A foundational primer. Journal of Mathematical Psychology, 44, 408-463.

Starns, J. J., \& Ratcliff, R. (2012). Age-related differences in diffusion model boundary optimality with both trial-limited and time-limited tasks. Psychonomic Bulletin 83 Review, 19(1), 139145.

Staugaard, C. F., Petersen, A., \& Vangkilde, S. (2016, November). Eccentricity effects in vision and attention. Neuropsychologia, 92, 69-78. Retrieved 2020-09-23, from https://www.ncbi .nlm.nih.gov/pmc/articles/PMC5127899/ doi: 10.1016/j.neuropsychologia.2016.06.020

Stephan, K. E., Penny, W. D., Daunizeau, J., Moran, R. J., \& Friston, K. J. (2009). Bayesian model selection for group studies. Neuroimage, 46(4), 1004-1017.

Stone, M. (1960). Models for choice-reaction time. Psychometrika, 25, 251-260.

Strasburger, H., Rentschler, I., \& Jüttner, M. (2011, May). Peripheral vision and pattern recognition: A review. Journal of Vision, 11(5), 13-13. Retrieved 2020-04-09, from http://jov. arvojournals.org/article.aspx?articleid=2191825 (Publisher: The Association for Research in Vision and Ophthalmology) doi: 10.1167/11.5.13

Stroop, J. R. (1935). Studies of interference in serial verbal reactions. Journal of Experimental 
Psychology, 18(6), 643-662. doi: 10.1037/h0054651

Ter Braak, C. J. (2006). A markov chain monte carlo version of the genetic algorithm differential evolution: easy bayesian computing for real parameter spaces. Statistics and Computing, 16(3), 239-249.

Tillman, G., Strayer, D., Eidels, A., \& Heathcote, A. (2017). Modeling cognitive load effects of conversation between a passenger and driver. Attention, Perception, $\mathcal{E}$ Psychophysics, 79(6), $1795-1803$.

Turner, B. M., Forstmann, B. U., Wagenmakers, E.-J., Brown, S. D., Sederberg, P. B., \& Steyvers, M. (2013). A Bayesian framework for simultaneously modeling neural and behavioral data. NeuroImage, 72, 193-206.

Turner, B. M., Rodriguez, C. A., Norcia, T. M., McClure, S. M., \& Steyvers, M. (2016). Why more is better: Simultaneous modeling of eeg, fmri, and behavioral data. NeuroImage, 128, $96-115$.

Turner, B. M., \& Sederberg, P. B. (2014). A generalized, likelihood-free method for posterior estimation. Psychonomic bulletin \& review, 21(2), 227-250.

Turner, B. M., Sederberg, P. B., Brown, S. D., \& Steyvers, M. (2013). A method for efficiently sampling from distributions with correlated dimensions. Psychological Methods, 18, 368-384.

Turner, B. M., Van Maanen, L., \& Forstmann, B. U. (2015). Informing cognitive abstractions through neuroimaging: The neural drift diffusion model. Psychological Review, 122, 312336.

Turner, B. M., Wang, T., \& Merkle, E. C. (2017). Factor analysis linking functions for simultaneously modeling neural and behavioral data. NeuroImage, 153, 28-48.

Tversky, A., \& Kahneman, D. (1974). Judgment under uncertainty: Heuristics and biases. Science, $185(4157), 1124-1131$.

Ulrich, R., Schröter, H., Leuthold, H., \& Birngruber, T. (2015, May). Automatic and controlled stimulus processing in conflict tasks: Superimposed diffusion processes and delta functions. Cognitive Psychology, 78, 148-174. doi: 10.1016/j.cogpsych.2015.02.005

Umiltà, C., Rubichi, S., \& Nicoletti, R. (1999, May). Facilitation and interference components in the Simon effect. Archives Italiennes De Biologie, 137(2-3), 139-149. 
Usher, M., \& McClelland, J. L. (2001, July). The time course of perceptual choice: the leaky, competing accumulator model. Psychological Review, 108(3), 550-592. doi: 10.1037/0033 $-295 x .108 .3 .550$

van den Wildenberg, W. P., Wylie, S. A., Forstmann, B. U., Burle, B., Hasbroucq, T., \& Ridderinkhof, K. R. (2010, December). To Head or to Heed? Beyond the Surface of Selective Action Inhibition: A Review. Frontiers in Human Neuroscience, 4. Retrieved 202009-22, from https://www.ncbi.nlm.nih.gov/pmc/articles/PMC3004391/ doi: 10.3389/ fnhum.2010.00222

van Ravenzwaaij, D., Brown, S., \& Wagenmakers, E.-J. (2011). An integrated perspective on the relation between response speed and intelligence. Cognition, 119(3), 381-393.

van Ravenzwaaij, D., Dutilh, G., \& Wagenmakers, E.-J. (2012). A diffusion model decomposition of the effects of alcohol on perceptual decision making. Psychopharmacology, 219(4), 10171025.

van Ravenzwaaij, D., \& Oberauer, K. (2009). How to use the diffusion model: Parameter recovery of three methods: Ez, fast-dm, and dmat. Journal of Mathematical Psychology, 53(6), 463-473.

Wallace, R. J. (1971, June). S-R compatibility and the idea of a response code. Journal of Experimental Psychology, 88(3), 354-360. doi: 10.1037/h0030892

White, C. N., Ratcliff, R., \& Starns, J. J. (2011, December). Diffusion models of the flanker task: discrete versus gradual attentional selection. Cognitive Psychology, 63(4), 210-238. doi: 10.1016/j.cogpsych.2011.08.001

White, C. N., Servant, M., \& Logan, G. D. (2018). Testing the validity of conflict drift-diffusion models for use in estimating cognitive processes: A parameter-recovery study. Psychonomic Bulletin \& Review, 25(1), 286-301. doi: 10.3758/s13423-017-1271-2

Wühr, P., \& Biebl, R. (2011). The role of working memory in spatial sr correspondence effects. Journal of Experimental Psychology: Human Perception and Performance, 37(2), 442.

Yeh, Y. Y., \& Eriksen, C. W. (1984, May). Name codes and features in the discrimination of letter forms. Perception \& Psychophysics, 36(3), 225-233. Retrieved 2020-05-28, from https://doi.org/10.3758/BF03206363 doi: 10.3758/BF03206363 

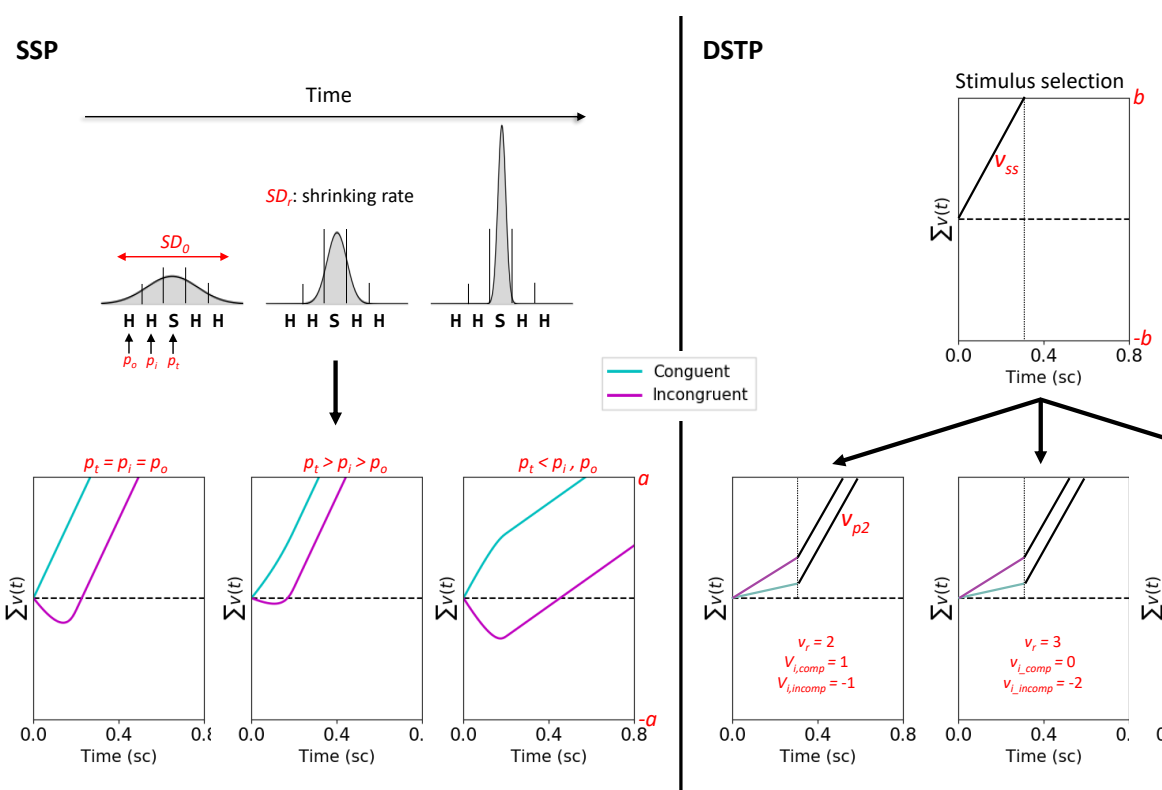

Figure 1. A graphical representation of the shrinking spotlight model (SSP) and the dual-stage twophase model (DSTP), and why these models do not naturally extend to disentangling facilitation and interference effects. To better illustrate the impact of the perceptual input of each flanker item on decision formation within SSP, we dissociated the perceptual input of inner $\left(p_{i}\right)$ and outer $\left(p_{o}\right)$ flankers. Facilitation requires larger $p_{i}$ and $p_{o}$ compared to the perceptual input of the central target $p_{t}$, which appears implausible in light of the well-established decrease in visual acuity as eccentricity increases. For DSTP, different combinations of the drift rate for the task-relevant information $v_{t}$, drift rate for the task-irrelevant information in compatible trials $v_{\text {comp }}$, and drift rate for the task-irrelevant information in incompatible trials $v_{\text {incomp }}$ produce identical drift rate dynamics in phase 1 of response selection, making it impossible to disentangle facilitation and interference effects. Remaining SSP parameters: upper decision bound $a$ corresponding to the correct response, initial spotlight width $S D_{0}$, spotlight shrinking rate $S D_{r}$. Remaining DSTP parameters: upper response selection bound $a$ corresponding to the correct response, upper stimulus selection bound $b$ corresponding to the selection of the central target, drift rate of response selection in phase $2 v_{p 2}$. 


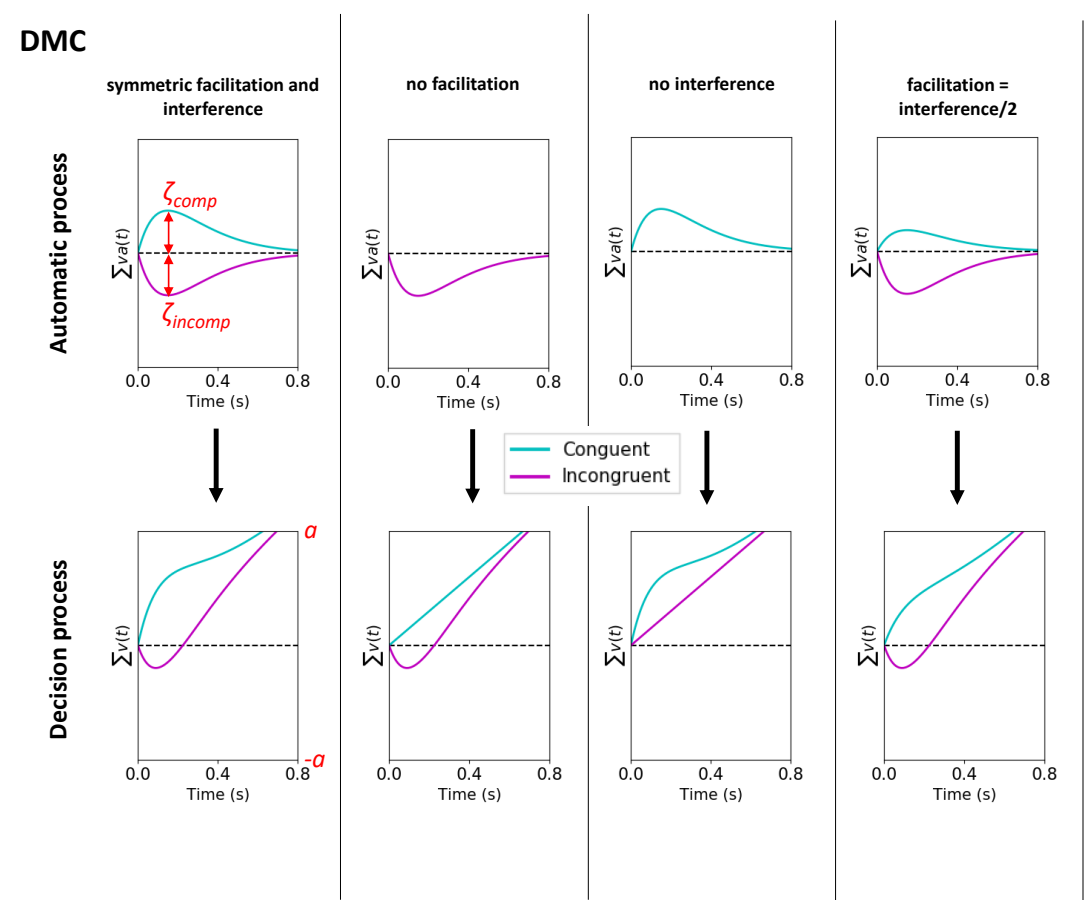

Figure 2. A graphical representation of the diffusion model for conflict tasks (DMC, column 1), and our extension of the model to disentangle facilitation and interference effects (columns 2-4). The extension consists in dissociating the amplitude of the automatic activation $\zeta$ in compatible $\left(\zeta_{\text {comp }}\right)$ and incompatible trials $\left(\zeta_{\text {incomp }}\right)$. Column 2 shows dynamics of decision formation when $\zeta_{c o m p}=0$, resulting in no facilitation. Column 3 shows decision formation when $\zeta_{\text {incomp }}=0$, resulting in no interference. Finally, column 4 shows decision formation when $\zeta_{\text {comp }}=\zeta_{\text {incomp }} / 2$, resulting in a facilitation effect equal to half the interference effect. The upper decision bound $a$ corresponds to the correct response. 

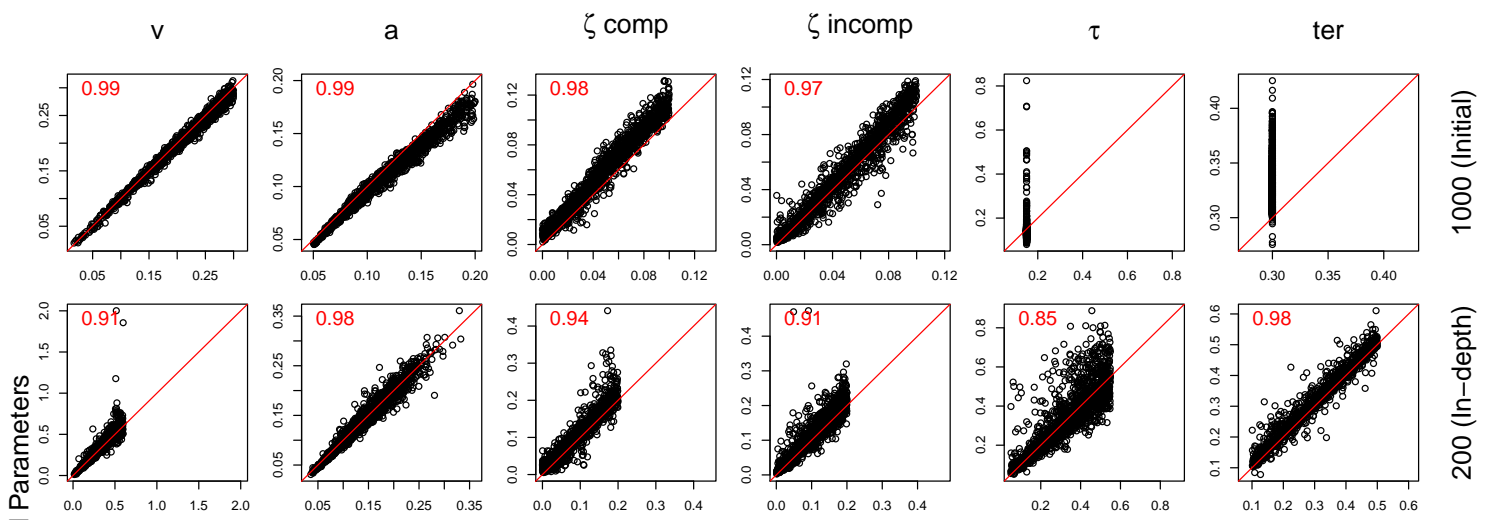

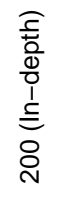
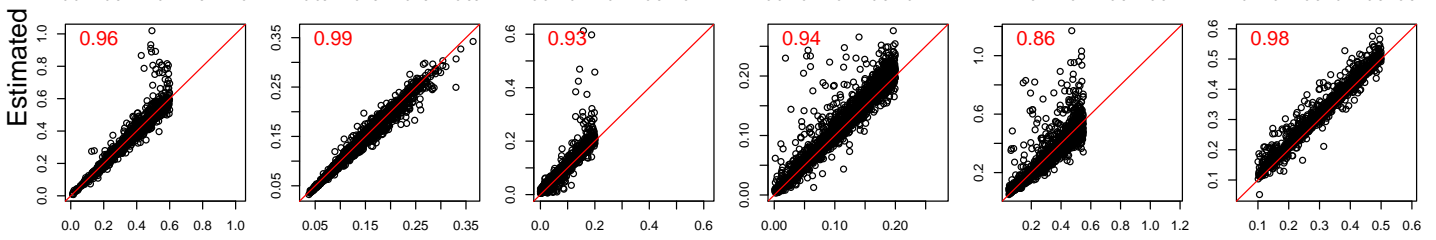

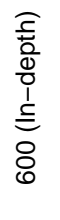
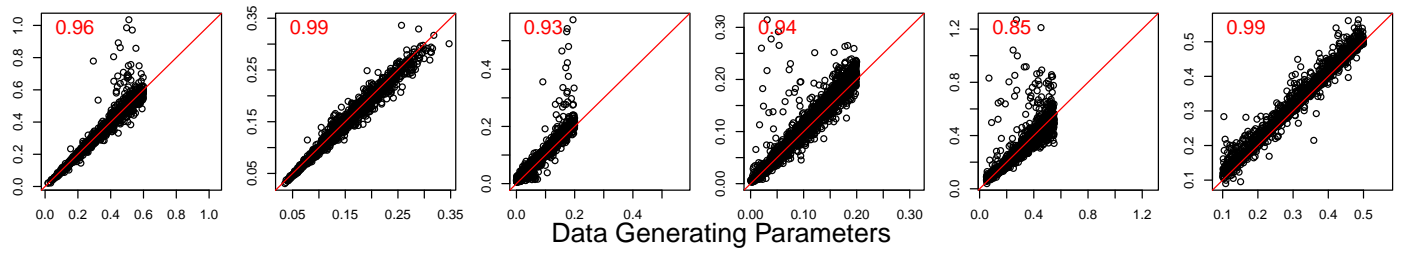

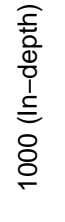

Figure 3. Parameter recovery assessment of our extended DMC framework. The x-axis displays the data generating values and the y-axis displays the estimated posterior means, with each data point representing a different simulated data set. The red line reflects perfect recovery, and the red number in the top-left corner of each plot displays the correlation coefficient between the generated and estimated values across data sets. Each column displays a different parameter, and each row displays a different recovery simulation. 


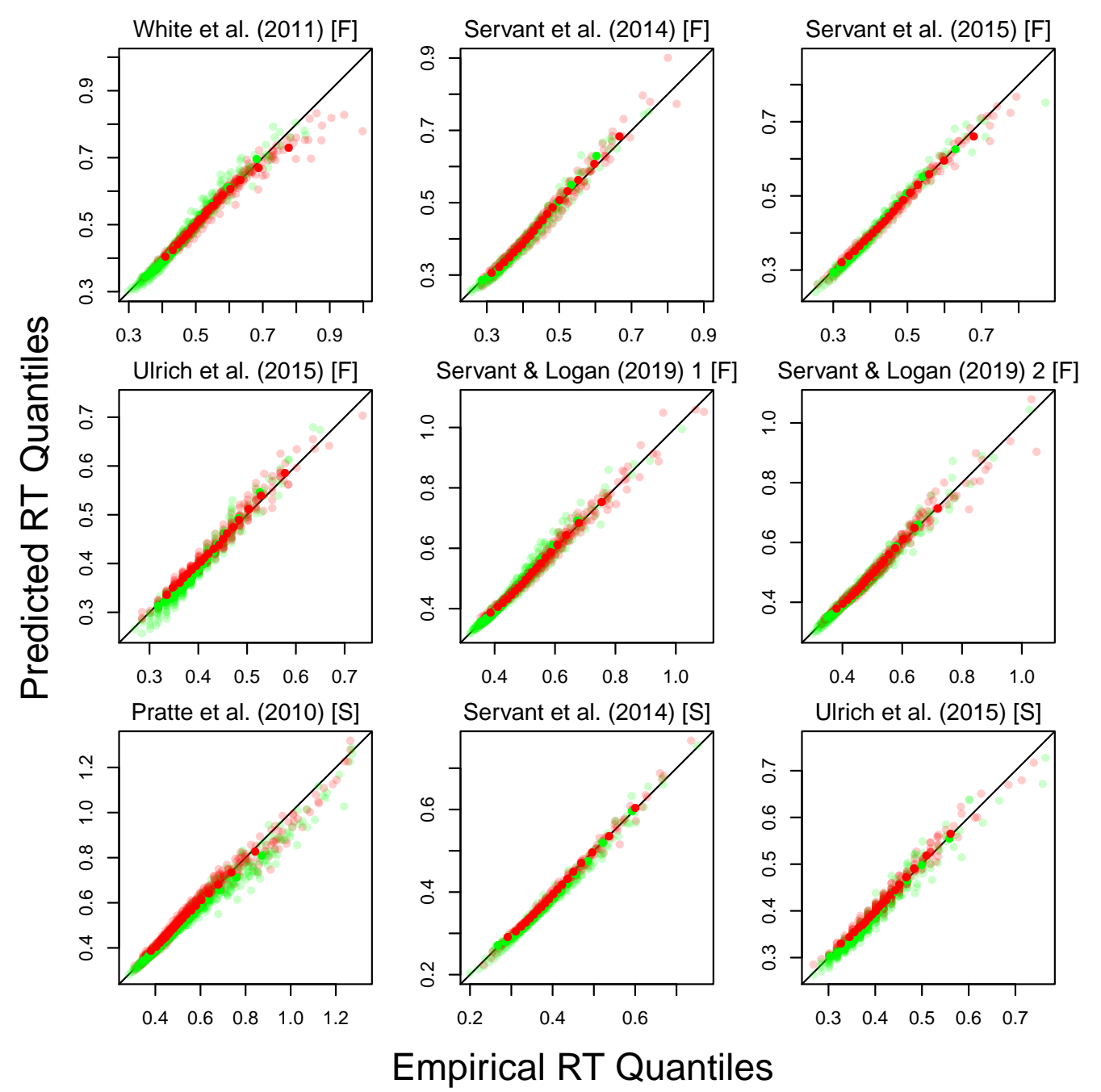

Figure 4. Goodness-of-fit assessment for our extended DMC framework on each of the 9 data sets included in our study. Each plot shows a different data set, with $[\mathrm{F}]$ reflecting flanker data sets, and $[\mathrm{S}]$ reflecting Simon data sets. The x-axis displays the empirical response tie quantiles, and the y-xis displays the model predicted response time quantiles based on the posterior mode, with the black line reflecting perfect fit. Green dots displays the quantiles for correct responses in compatible trials, whereas red dots display quantiles for correct responses in incompatible trials, with 19 quantiles being displays ( 0.05 to 0.95 , in increments of 0.05 ). The highly transparent dots reflect the fits of all individual subjects, whereas the solid coloured dots reflect the group-averaged fits based on averaging the empirical and predicted quantiles across participants. 

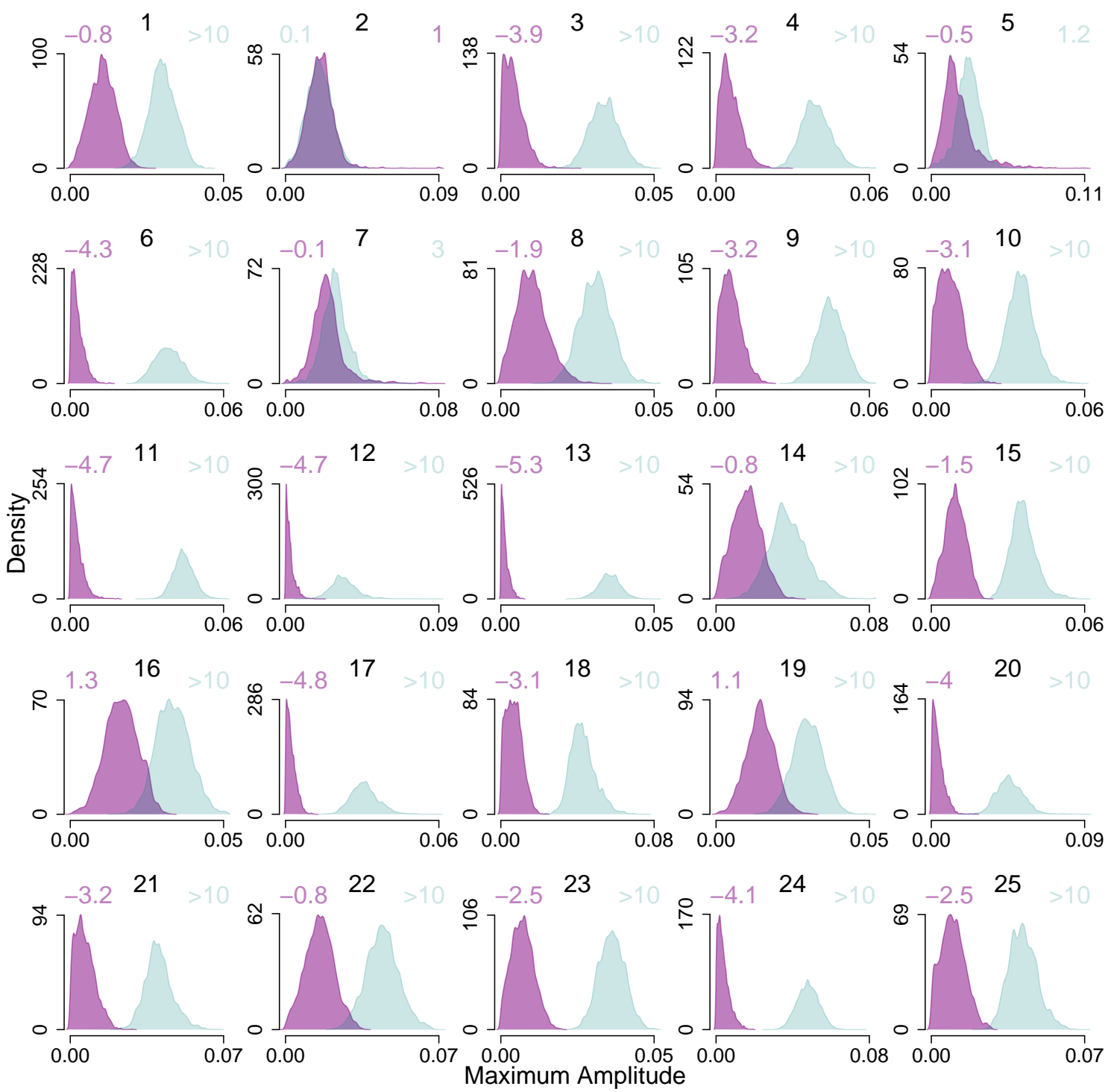

Figure 5. Results for the White et al. (2011) data set. Each plot within the figure displays a different participant, with the x-axis of each plot displaying the value of the $\zeta$ parameter, and the $\mathrm{y}$-axis displaying the estimated posterior density for those values. In each plot, the posterior distributions for the $\zeta_{\text {comp }}$ and $\zeta_{\text {incomp }}$ parameters are in purple and aqua, respectively. The numbers in purple and aqua display the log-Bayes factor for the $\zeta_{\text {comp }}$ and $\zeta_{\text {incomp }}$ parameters, respectively, being greater than zero. The placement of these numbers (top-left vs. top-right) were based on the posterior means for the parameters, with the parameter that had the higher posterior mean being placed on the right. 

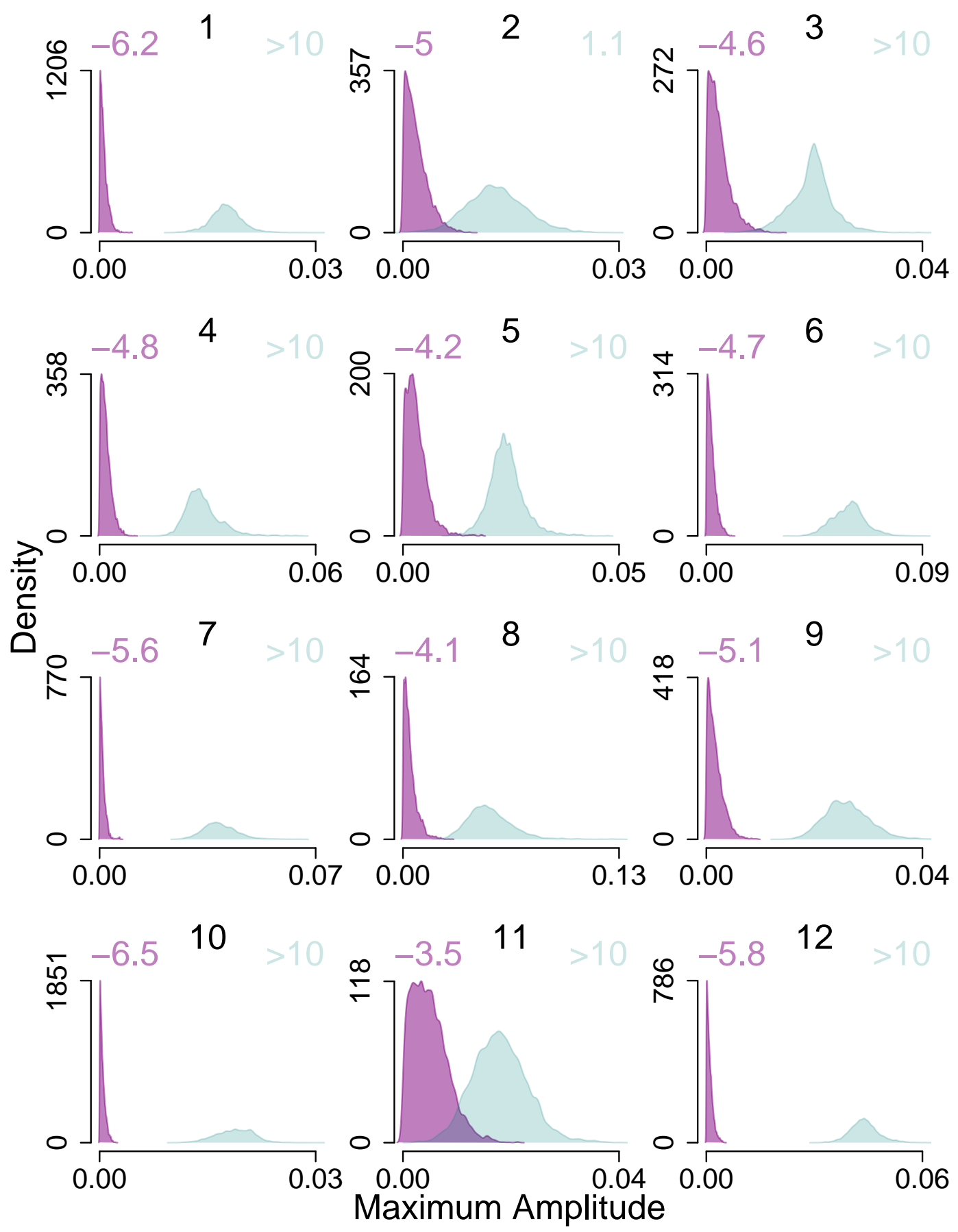

Figure 6. Results for the Servant et al. (2014) flanker data set. Each plot within the figure displays a different participant, with the x-axis of each plot displaying the value of the $\zeta$ parameter, and the $y$-axis displaying the estimated posterior density for those values. In each plot, the posterior distributions for the $\zeta_{c o m p}$ and $\zeta_{\text {incomp }}$ parameters are in purple and aqua, respectively. The numbers in purple and aqua display the log-Bayes factor for the $\zeta_{\text {comp }}$ and $\zeta_{\text {incomp }}$ parameters, respectively, being greater than zero. The placement of these numbers (top-left vs. top-right) were based on the posterior means for the parameters, with the parameter that had the higher posterior mean being placed on the right. 

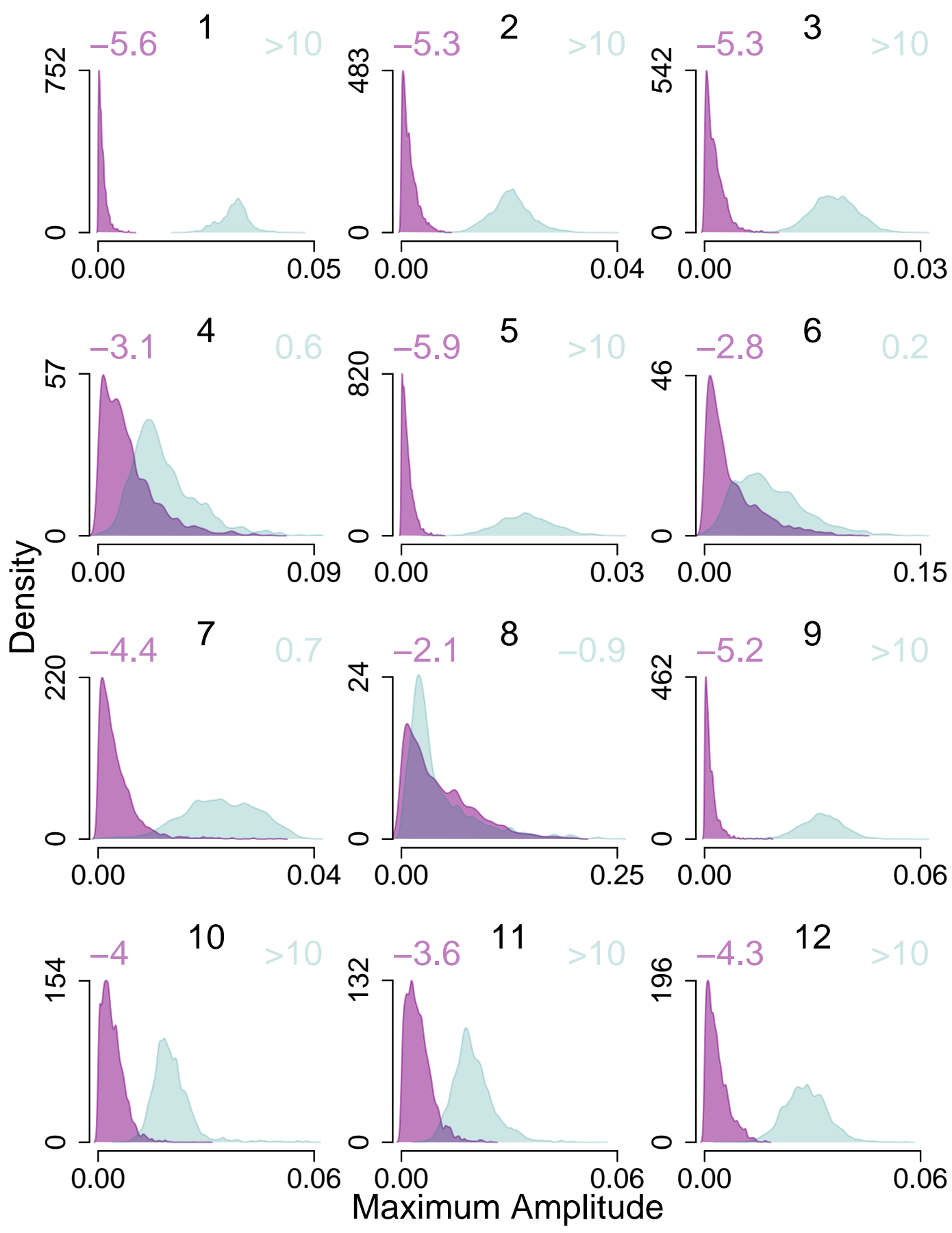

Figure 7. Results for the Servant et al. (2015) data set. Each plot within the figure displays a different participant, with the x-axis of each plot displaying the value of the $\zeta$ parameter, and the y-axis displaying the estimated posterior density for those values. In each plot, the posterior distributions for the $\zeta_{\text {comp }}$ and $\zeta_{\text {incomp }}$ parameters are in purple and aqua, respectively. The numbers in purple and aqua display the log-Bayes factor for the $\zeta_{\text {comp }}$ and $\zeta_{\text {incomp }}$ parameters, respectively, being greater than zero. The placement of these numbers (top-left vs. top-right) were based on the posterior means for the parameters, with the parameter that had the higher posterior mean being placed on the right. 

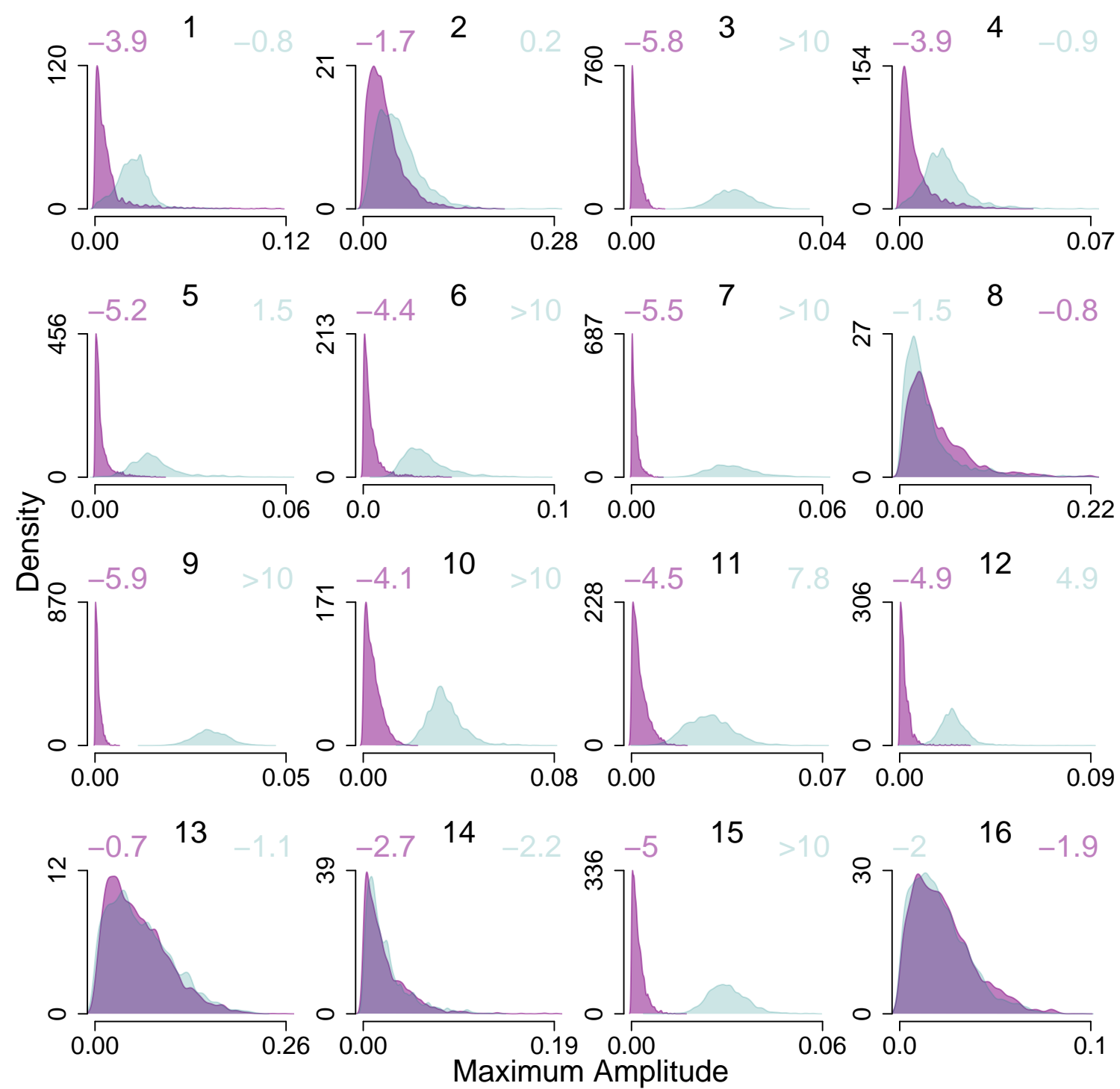

Figure 8. Results for the Ulrich et al. (2015) flanker data set. Each plot within the figure displays a different participant, with the x-axis of each plot displaying the value of the $\zeta$ parameter, and the $\mathrm{y}$-axis displaying the estimated posterior density for those values. In each plot, the posterior distributions for the $\zeta_{c o m p}$ and $\zeta_{\text {incomp }}$ parameters are in purple and aqua, respectively. The numbers in purple and aqua display the log-Bayes factor for the $\zeta_{\text {comp }}$ and $\zeta_{\text {incomp }}$ parameters, respectively, being greater than zero. The placement of these numbers (top-left vs. top-right) were based on the posterior means for the parameters, with the parameter that had the higher posterior mean being placed on the right. 

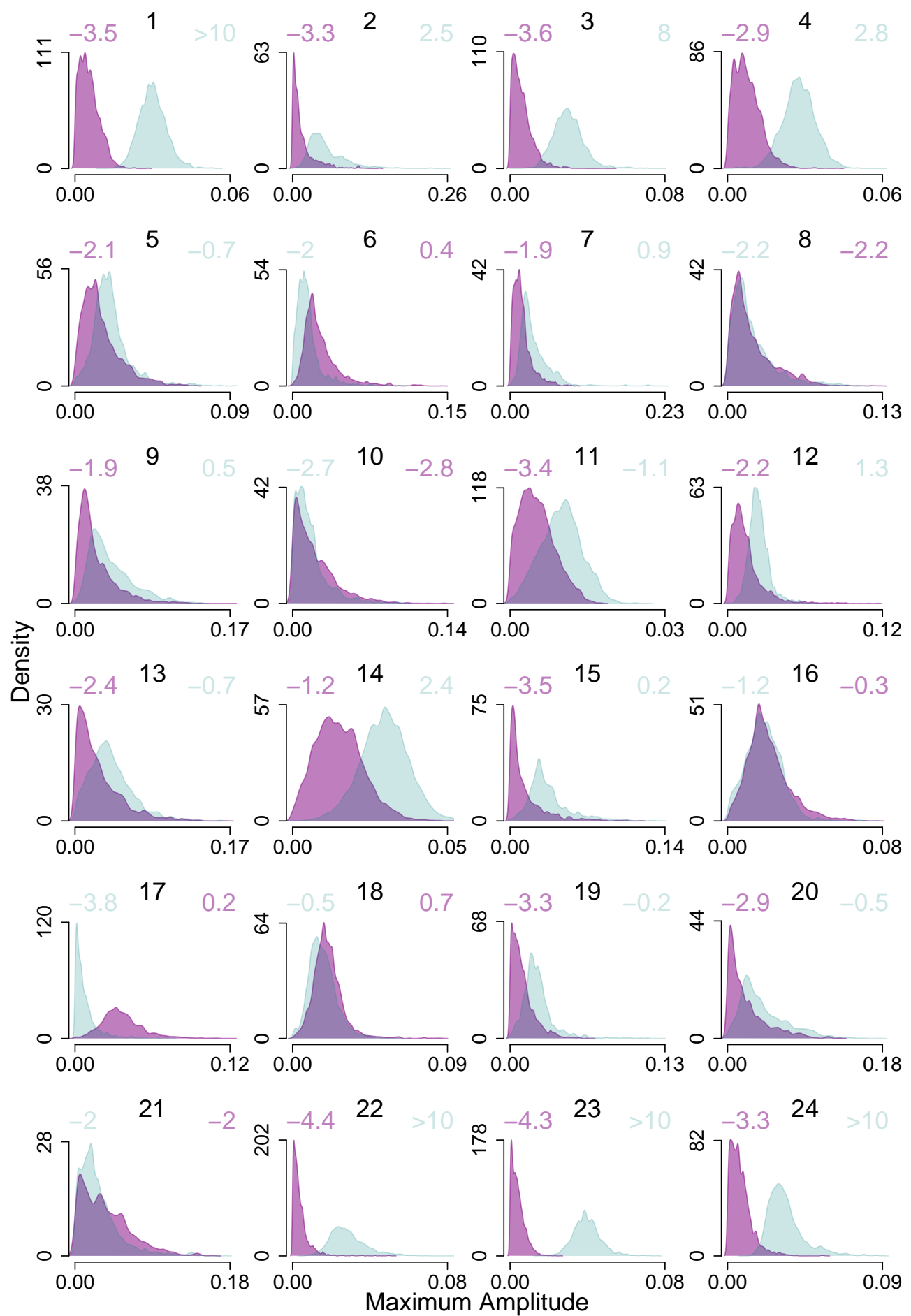

Figure 9. Results for the first Servant \& Logan (2019) data set. Each plot within the figure displays a different participant, with the $\mathrm{x}$-axis of each plot displaying the value of the $\zeta$ parameter, and the $y$-axis displaying the estimated posterior density for those values. In each plot, the posterior distributions for the $\zeta_{c o m p}$ and $\zeta_{\text {incomp }}$ parameters are in purple and aqua, respectively. The numbers in purple and aqua display the log-Bayes factor for the $\zeta_{\text {comp }}$ and $\zeta_{\text {incomp }}$ parameters, respectively, being greater than zero. The placement of these numbers (top-left vs. top-right) were based on the posterior means for the parameters, with the parameter that had the higher posterior mean being placed on the right. 

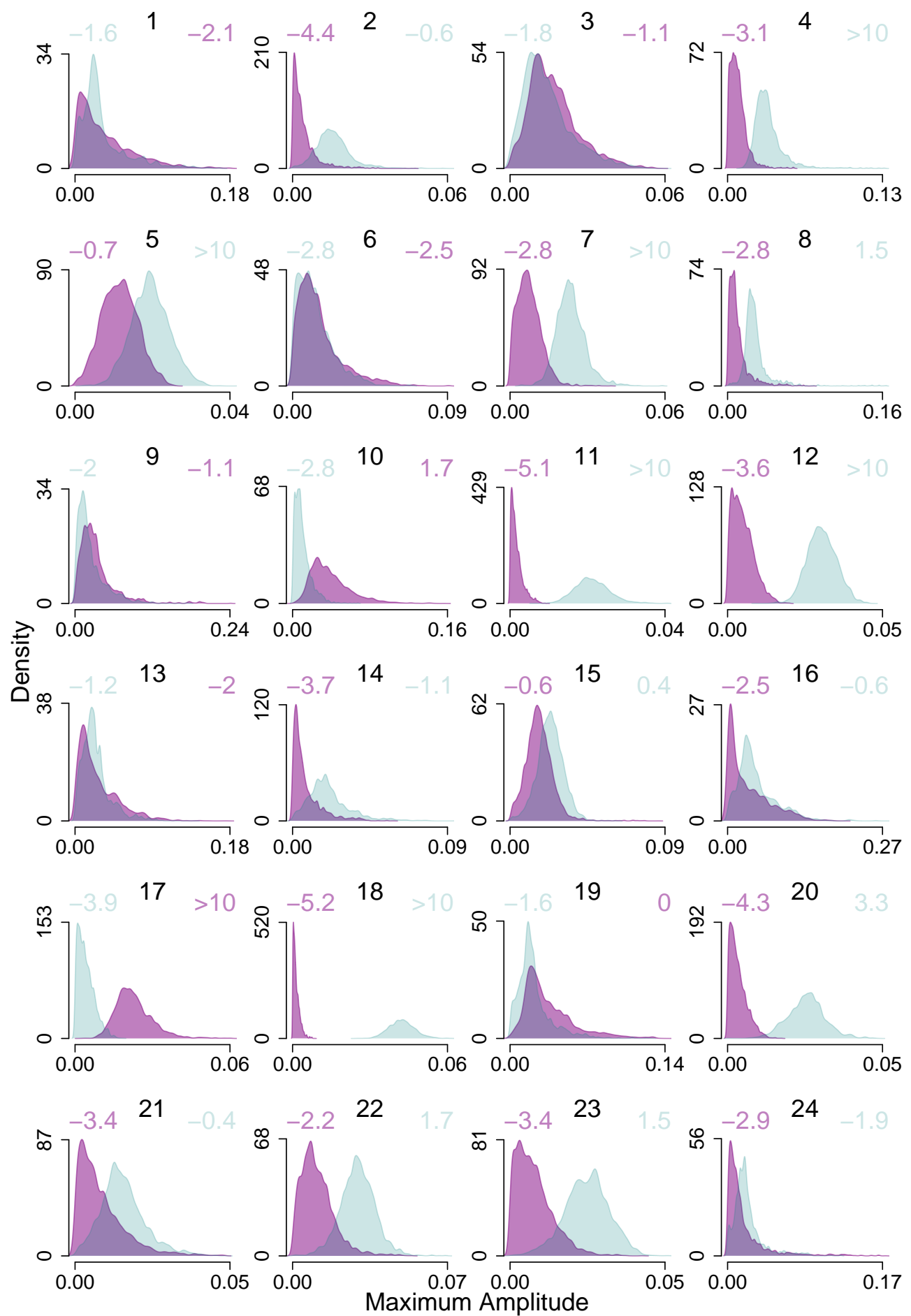

Figure 10. Results for the second Servant \& Logan (2019) data set. Each plot within the figure displays a different participant, with the x-axis of each plot displaying the value of the $\zeta$ parameter, and the y-axis displaying the estimated posterior density for those values. In each plot, the posterior distributions for the $\zeta_{c o m p}$ and $\zeta_{\text {incomp }}$ parameters are in purple and aqua, respectively. The numbers in purple and aqua display the log-Bayes factor for the $\zeta_{\text {comp }}$ and $\zeta_{\text {incomp }}$ parameters, respectively, being greater than zero. The placement of these numbers (top-left vs. top-right) were based on the posterior means for the parameters, with the parameter that had the higher posterior mean being placed on the right. 

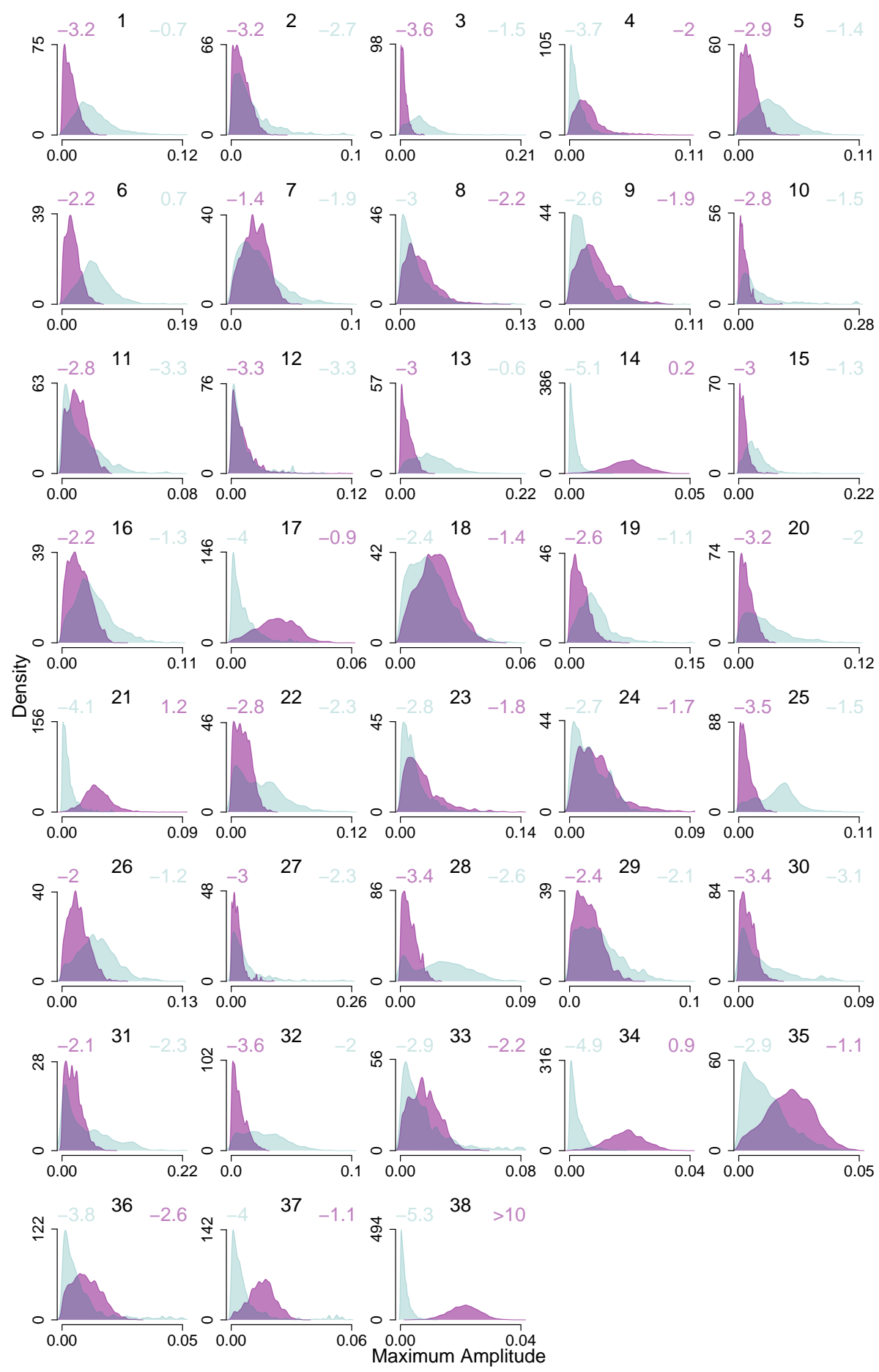

Figure 11. Results for the Pratte et al. (2010) data set. Each plot within the figure displays a different participant, with the $\mathrm{x}$-axis of each plot displaying the value of the $\zeta$ parameter, and the $\mathrm{y}$-axis displaying the estimated posterior density for those values. In each plot, the posterior distributions for the $\zeta_{c o m p}$ and $\zeta_{\text {incomp }}$ parameters are in purple and aqua, respectively. The numbers in purple and aqua display the log-Bayes factor for the $\zeta_{\text {comp }}$ and $\zeta_{\text {incomp }}$ parameters, respectively, being greater than zero. The placement of these numbers (top-left vs. top-right) were based on the posterior means for the parameters, with the parameter that had the higher posterior mean being placed on the right. 

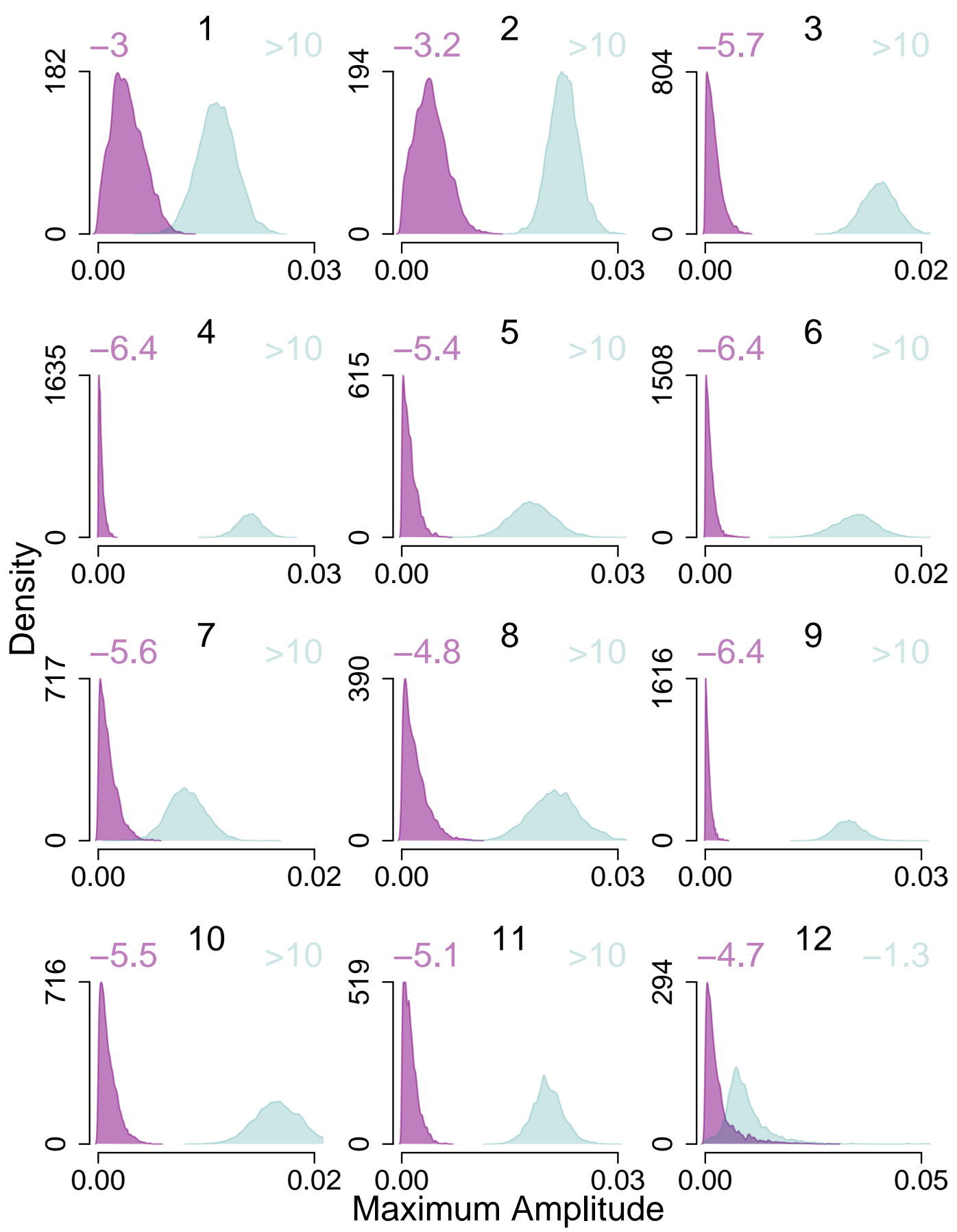

Figure 12. Results for the Servant et al. (2014) Simon data set. Each plot within the figure displays a different participant, with the x-axis of each plot displaying the value of the $\zeta$ parameter, and the y-axis displaying the estimated posterior density for those values. In each plot, the posterior distributions for the $\zeta_{\text {comp }}$ and $\zeta_{\text {incomp }}$ parameters are in purple and aqua, respectively. The numbers in purple and aqua display the log-Bayes factor for the $\zeta_{\text {comp }}$ and $\zeta_{\text {incomp }}$ parameters, respectively, being greater than zero. The placement of these numbers (top-left vs. top-right) were based on the posterior means for the parameters, with the parameter that had the higher posterior mean being placed on the right. 

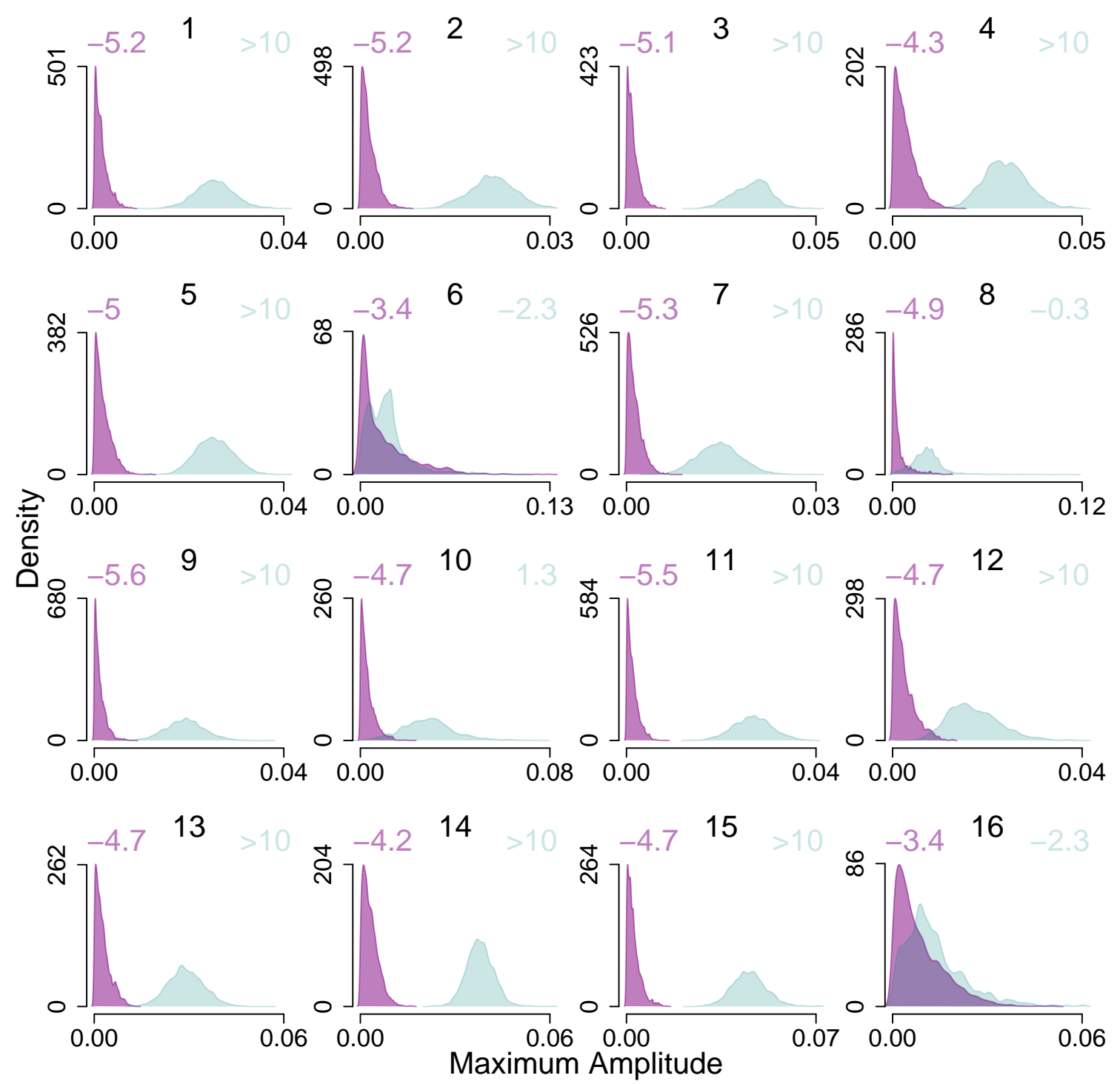

Figure 13. Results for the Ulrich et al. (2015) Simon data set. Each plot within the figure displays a different participant, with the x-axis of each plot displaying the value of the $\zeta$ parameter, and the $\mathrm{y}$-axis displaying the estimated posterior density for those values. In each plot, the posterior distributions for the $\zeta_{c o m p}$ and $\zeta_{\text {incomp }}$ parameters are in purple and aqua, respectively. The numbers in purple and aqua display the log-Bayes factor for the $\zeta_{\text {comp }}$ and $\zeta_{\text {incomp }}$ parameters, respectively, being greater than zero. The placement of these numbers (top-left vs. top-right) were based on the posterior means for the parameters, with the parameter that had the higher posterior mean being placed on the right. 